\title{
Design study of compact medical accelerator using superconducting rf quadrupole for boron neutron capture therapy
}

\author{
Ryo Katayama, Eiji Kako®, Seiya Yamaguchi, Shinichiro Michizono®, and Kensei Umemori® \\ High Energy Accelerator Research Organization (KEK), \\ 1-1 Oho, Tsukuba, Ibaraki 305-0801, Japan \\ Yasuhiro Kondo \\ Japan Atomic Energy Agency (JAEA), Tokai, Naka, Ibaraki, 319-1195, Japan
}

(Received 15 February 2021; accepted 3 February 2022; published 18 February 2022)

\begin{abstract}
We investigated the feasibility on the application of a superconducting radio frequency (SRF) niobium cavity to an accelerator-based neutron source for boron neutron capture therapy (BNCT). Neutron source is the key component of BNCT and adopting rf-linac based neutron source realizes a medical care system sufficient to be compact, which can be installed in a hospital and to generate intensive neutron yields that the BNCT requires. However, it is still desirable to improve the efficiency of input power on neutron yields and the achievable accelerate field gradient. SRF accelerator technology potentially allows us to enhance the performance because of its prominent lower ohmic loss and higher sustainable accelerating fields. This paper presents a first feasibility study on the application of a SRF niobium cavity to an accelerator-based neutron source for BNCT, assuming that a superconducting radio frequency quadrupole (SC-RFQ) composed of pure bulk niobium at $4.2 \mathrm{~K}$ accelerates the proton/deuteron beams to a beryllium or lithium target for the neutron production of BNCT via ${ }^{7} \mathrm{Li}(\mathrm{p}, \mathrm{n}){ }^{7} \mathrm{Be},{ }^{9} \mathrm{Be}(\mathrm{p}, \mathrm{n}){ }^{9} \mathrm{~B}$, or ${ }^{9} \mathrm{Be}(\mathrm{d}, \mathrm{n}){ }^{10} \mathrm{~B}$. The following beam parameters were used: beam energy of $2.5 \mathrm{MeV}$ (for Li target)/5 MeV (for Be target), ion source current (50 keV, CW $30 \mathrm{~mA}$ ), normalized beam emittance of $0.02 \mathrm{~cm}$ mrad, and resonance frequency of $325 \mathrm{MHz}$ (for proton)/162.5 MHz (for deuteron). Based on these conditions, we evaluated the feasibility on the following three criteria: comparison of the cooling capacity of the refrigerator to the amount of heat, power consumption of AC, and size of the BNCT system. First, we evaluated the amount of heat generated in a cryomodule by adding the ohmic loss of SC-RFQ $Q_{\mathrm{rf}}$, beam losses in SC-RFQ $Q_{\mathrm{b}}$, heat penetration into the cryomodule $Q_{\text {ext }}$, and beam losses of molecular ion beams and poor quality beams emitted from the ion-source at/near the RFQ entrance $Q_{\text {emit. }}$. In this study, we typically regarded $Q_{\text {ext }}$ as $20 \mathrm{~W}$ at $4.2 \mathrm{~K}$ and considered a new low-energy beam transport system that can suppress $Q_{\text {emit }}$ to 0 . In addition, $Q_{\mathrm{rf}}$ and $Q_{\mathrm{b}}$ were numerically evaluated by beam simulation and electromagnetic calculation. The obtained results revealed that the sum of the heat amounts could be sufficiently suppressed below the typical cooling capacity of a commercially available helium refrigerator. Second, we compared the ac power consumption of BNCT between a conventional and SC-BNCT systems, which indicated that the BNCT system adopting the SRF cavity effectively reduced the ac power consumption of SC-BNCT by almost $1 / 4$ times. Third, the length of the SC-RFQ could be shortened by adjusting the peak surface E-field, as compared to conventional existing RFQs such as J-PARC, SNS, and IFMIF. Eventually, this study demonstrated that the application of the SRF cavity for the rf-linac-based neutron source of BNCT is feasible, and thus provides a foundation for the future development of design for next-generation BNCT systems.
\end{abstract}

DOI: 10.1103/PhysRevAccelBeams.25.021601

\section{INTRODUCTION}

Boron neutron capture therapy (BNCT) is a promising

"ryo.katayama@kek.jp

Published by the American Physical Society under the terms of the Creative Commons Attribution 4.0 International license. Further distribution of this work must maintain attribution to the author(s) and the published article's title, journal citation, and DOI. radiotherapy treatment method for intractable cancer, where ${ }^{10} \mathrm{~B}$-compound that accumulates only in the cancer cells is administered to patients and neutron beams are irradiated onto them. In this situation, the nuclear reaction ${ }^{10} \mathrm{~B}(\mathrm{n}, \alpha)^{7} \mathrm{Li}$ occurs only in the cancer cells. As the track ranges of the two emitted particles are too short to exceed the size of a typical cell, both alpha-rays and ${ }^{7} \mathrm{Li}$ emitted 
from this region selectively kill the cancer cells without damaging the surrounding normal cells. In this context, the neutron source is the most important tool for realizing the BNCT system. For the treatment of BNCT, the more intense the neutron beams are, the shorter the time for the treatment is, which can relieve the burden of patients. Although a nuclear reactor has been conventionally used as a neutron source for clinical trials of BNCT, the construction of a nuclear reactor is not feasible for a hospital owing to limitations in space and difficulties in its appropriate operation and maintenance. In addition, users cannot avoid treating nuclear fuels. Thus, as an alternative, an accelerator-based neutron source has been developed and put into practice in recent years. The BNCT system using an accelerator-based neutron source is sufficiently compact for installation in a hospital and has the potential to become a standard method for intractable cancer therapy.

The neutrons in the accelerator-based neutron source for BNCT are generated from a nuclear reaction in which proton/deuteron beams with a kinetic energy greater than several $\mathrm{MeV}$ are injected into a target material. In particular, there are two standard materials: lithium and beryllium. The neutrons for a lithium target can be effectively produced from a nuclear reaction $\mathrm{p}(\mathrm{Li}, \mathrm{Be}) \mathrm{n}$ that has a resonance peak of neutron production cross section around 2.5 MeV. For instance, the national cancer center in Japan has developed a BNCT system using an accelerator-based neutron source that injects proton beams $(20 \mathrm{~mA}, 2.5 \mathrm{MeV})$ into a lithium target [1] to produce an adequate number of neutrons for the medical treatment. On the contrary, nuclear reactions for ${ }^{9} \mathrm{Be}(\mathrm{p}, \mathrm{n}){ }^{9} \mathrm{~B}$ and ${ }^{9} \mathrm{Be}(\mathrm{d}, \mathrm{n}){ }^{10} \mathrm{~B}$ do not exhibit a resonance peak in the neutron production cross section to enhance the neutron yield per unit beam current. Thus, the number of production neutrons and the emitted neutron energy increased with the incident beam energy. For instance, the Ibaraki Neutron Medical Research Center has developed a BNCT system (i-BNCT) for the linacbased neutron source wherein the proton beams (avg $5 \mathrm{~mA}$, $8 \mathrm{MeV}$ ) are injected into a Beryllium target [2] to produce a considerable number of neutrons for the medical treatment. According to a prior research [3], the number of neutrons produced per unit beam current reduces to approximately one-third when the proton beam energy changes from $8 \mathrm{MeV} \rightarrow$ to $5 \mathrm{MeV}$. Therefore, the incident beam current on this case should be set at $15 \mathrm{~mA}$ for the medical treatment using BNCT. Similarly [3], the number of neutrons produced per unit beam current reduces to half when deuteron beams of $5 \mathrm{MeV}$ are used instead of $8-\mathrm{MeV}$ proton beams. Therefore, the incident beam current in this case should be set at $10 \mathrm{~mA}$ for the medical treatment using BNCT. The beam intensities determined by target materials, particle species, and the neutron yield necessary for the medical treatment of BNCT are summarized in Table I.

Several accelerators have been employed in acceleratorbased neutron sources developed for BNCT. The rf-linac is a superior particle accelerator in terms of its high electric
TABLE I. The required beam intensity for producing adequate number of neutrons for boron neutron capture therapy. The aim value of the neutron yield is determined by the accelerated particle, beam energy, beam current, and a target material (Be or Li). The National Cancer Center in Japan adopts the BNCT system with a beam intensity of (2.5 MeV, $20 \mathrm{~mA})$ and lithium target [1]. The i-BNCT project proceeding at Ibaraki, Japan, aims to develop a BNCT system with a beam intensity of $(8 \mathrm{MeV}$, $5 \mathrm{~mA}$ ) and Beryllium target [2].

\begin{tabular}{lcc}
\hline \hline Particle & Requirement of Beam Intensity & Target \\
\hline Proton & $2.5 \mathrm{MeV}, 20 \mathrm{~mA}$ & Lithium \\
Proton & $8 \mathrm{MeV}, 5 \mathrm{~mA}$ & Beryllium \\
Proton & $5 \mathrm{MeV}, 15 \mathrm{~mA}$ & Beryllium \\
Deuteron & $5 \mathrm{MeV}, 10 \mathrm{~mA}$ & Beryllium \\
\hline \hline
\end{tabular}

field gradient and discharge stability, thus allowing a BNCT system adopting an rf-linac-based neutron source to achieve both 5-10 MeV of particle energy and a total system size of less than $10 \mathrm{~m}$. Conventionally, the main accelerator component for the rf-linac-based neutron source is a rf resonant cavity. In general, the inner surfaces of the resonant cavities are coated with copper to reduce the rf surface resistance. Hereafter, the resonant cavity coated with copper for particle acceleration is referred as to "normal-conducting rf (NRF) cavity." The resonant cavity can store the electromagnetic wave received from a $\mathrm{rf}$ source and accelerate charged particles by using their electric field components. The electromagnetic fields stored in an NRF cavity induce rf currents on the surface and corresponds to a rf power loss owing to a surface resistance of several $\mathrm{m} \Omega$. The ohmic loss causes a heat emission of several $100 \mathrm{~kW}$ from the inner surfaces of the NRF cavity. Thus, the rf linac-based neutron source requires a powerful rf source for the BNCT system to overcome the ohmic loss of the NRF cavity (e.g., several 100-kW CW power klystrons), a huge water-cooling system for the NRF cavity and an rf source that increases both the installation and operation costs. Although a $10 \%-20 \%$ pulse operational management can be effective in reducing the operation cost, the neutron yield is diminished. In addition, the peak surface E-field of an NRF cavity is strongly constrained by the Kilpatrick discharge limit, and the maximum accelerated field gradient cannot be improved further.

Adopting rf-linac based neutron source using the NRF cavity realizes a medical care system sufficient to be compact and to generate intensive neutron yields for the treatment with BNCT; furthermore, it is still desirable to enhance the efficiency of input power on neutron yields and the achievable accelerator gradient from the financial point of view of cost reduction of BNCT system (e.g., capital investment in the early stage or ac power consumption during the treatment). Superconducting radio frequency (SRF) cavities potentially resolve the problems that a rflinac-based neutron source using NRF cavities have 


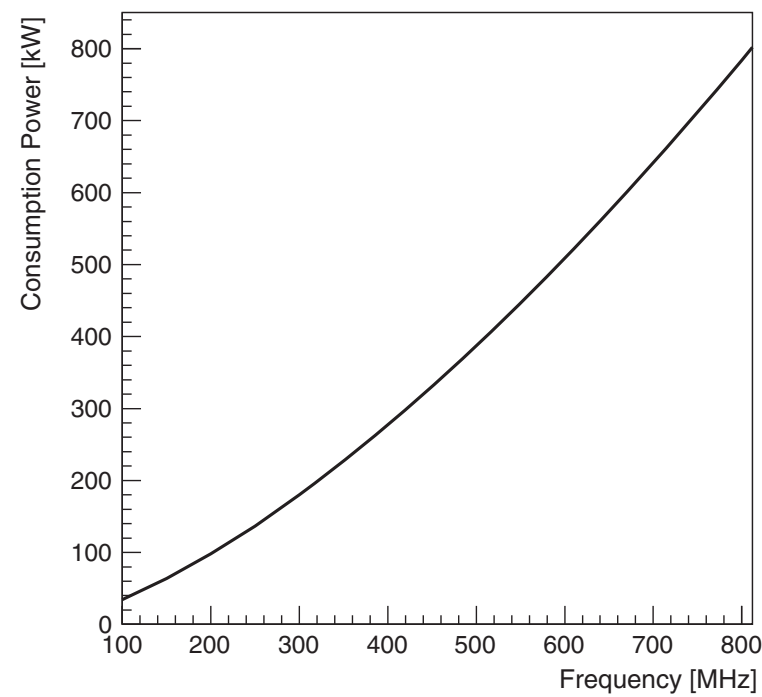

FIG. 1. The ohmic loss of $\mathrm{Cu}$ normal-conducting RFQ (NCRFQ) as a function of the resonant frequency. The ohmic loss was evaluated by the lumped circuit model of a RFQ length and intervane voltage of $3.1 \mathrm{~m}$ and $80 \mathrm{kV}$, respectively, similar to those of J-PARC. The ohmic loss of NC-RFQ was calculated using Eq. (A3).

because (1) the surface resistance of a SRF cavity is smaller than $1 / 100,000$ as compared to that of NRF cavities. Its prominent property effectively lightens the enormous water-cooling system and the high-power rf source. In particular, a 50-100 kW commercial solid-state amplifier can be used as a rf source instead of a conventional Klystron. Figures 1 and 2 show the amount of heat or

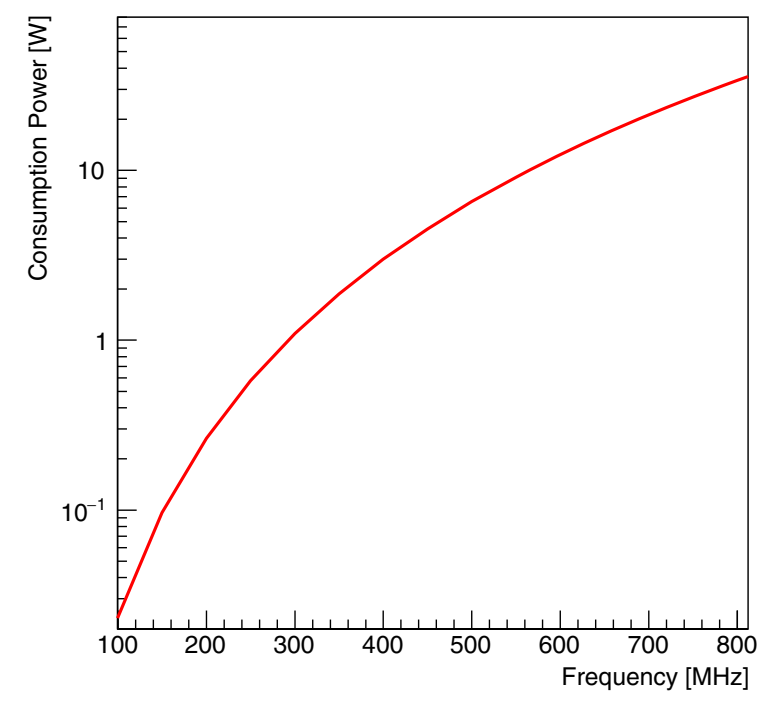

FIG. 2. The ohmic loss of Nb superconducting RFQ (SC-RFQ) as a function of the resonant frequency. The ohmic loss was evaluated by the lumped circuit model with a RFQ length and intervane voltage of $3.1 \mathrm{~m}$ and $80 \mathrm{kV}$, respectively, similar to those of J-PARC. The ohmic loss of SC-RFQ was calculated using Eq. (A6). the ohmic loss of a radio frequency quadrupole (RFQ) against its resonant frequency. Figure 1 depicts an NRF cavity coated with a copper thick-film; Fig. 2 displays a SRF cavity made of pure bulk niobium at $4.2 \mathrm{~K}$, where the resonant frequency and ohmic loss are represented on the horizontal and vertical axes, respectively. The ohmic loss or amount of heat for the NRF and SRF cavities were evaluated using the lumped circuit model of a four-vane cloverleaf geometry [see Eq. (A3) and Eq. (A6)]. Moreover, the ohmic loss of the NRF cavity directly correspond to its ac power consumption. On the other hand, the ohmic loss for the SRF cavity is required to be multiplied with the efficiency of the refrigerator cooling the SRF cavity. For instance, the cooling efficiency of a commercially available $4.2 \mathrm{~K}$ helium refrigerator is typically $0.6 \mathrm{~kW} / \mathrm{W}$ [4]. Thus, the corresponding ac power consumption of the NRF cavity in this case was estimated as $200 \mathrm{~kW}$ at $324 \mathrm{MHz}$, equivalent to the resonant frequency of J-PARC, and that of the SRF cavity was evaluated to be less than $2 \mathrm{~kW}$. Therefore, the ac power consumption or the heat-loading owing to ohmic loss can be significantly suppressed as compared to that of the normal conducting cavity. (2) The SRF cavity is not affected by the Kilpatrick discharge limit, and therefore, a relatively high electric-field strength can be selected to shorten the total length of the linac-based neutron source. For instance, the achievable peak surface E-field of $325 \mathrm{MHz}$ superconducting CH-DTL amounts to 2.5 Kilpatrick [5], which is 1.9 times greater than that of the 324-MHz J-PARC S-DTL [6].

This research conducted a first feasibility study on the application of a $\mathrm{Nb}$ SRF cavity on the rf-linac-based neutron source for BNCT, assuming that the neutron production for proton/deuteron beams were accelerated by a four-vane-type superconducting RFQ (SC-RFQ) made of $\mathrm{Nb}$ cooled at $4.2 \mathrm{~K}$. Hereafter, we refer to the BNCT system adopting a $4.2 \mathrm{~K} \mathrm{Nb}$ SC-RFQ as the "superconducting BNCT system (SC-BNCT)." Although the SRF cavity has great potential toward the design of the BNCT system, the following tasks are required to be evaluated.

(1) The amount of heat generated in a cryomodule must be lower than the cooling capacity of a commercially available helium refrigerator.

(2) The size of the SC-RFQ should be equal to or smaller than the existing normal-conducting RFQs.

(3) The mechanism of SC-BNCT system improving the ac power consumption as compared to the conventional BNCT system should be clarified.

RFQGen software was used to design the SC-RFQ and perform the beam simulation necessary for precisely evaluating its performance [7]. The following beam parameters were used in this study: beam energy of $2.5 \mathrm{MeV}$ (for Li target) $/ 5 \mathrm{MeV}$ (for Be target), ion source current (50 keV, CW $30 \mathrm{~mA}$ ), normalized beam emittance $0.02 \mathrm{~cm} \mathrm{mrad}$. 
Regarding resonant frequency, according to Figs. 1 and 2, the ohmic loss of RFQ improves as frequency becomes lower. On the other hand, in the situation of fixed length of RFQ as considered in Figs. 1 and 2, toward lower frequency, the number of cells is getting too low for a reasonable beam dynamics. Thus, we chose appropriate resonant frequencies of $325 \mathrm{MHz}$ (for $\mathrm{p}$ acceleration) and 162.5 MHz (for $\mathrm{d}$ acceleration). The Twiss parameters at the ion source position were selected to perfectly match the RFQ acceptance. As the Kilpatrick discharge limit was not empirically established for the SRF cavity, we selected the strength of the peak surface electric field $E_{p}$ - one of the input parameters of RFQGen - to be in the range from 35 to $60 \mathrm{MV} / \mathrm{m}$. In addition, the minimum radius aperture of the RFQGen was set at $0.32 \mathrm{~mm}$ (for proton) or $0.48 \mathrm{~mm}$ (for deuteron). A schematic of rf linac-based neutron sources assumed in this study is presented in Fig. 4. Moreover, the charge neutralization effect during traveling in low-energy beam transport (LEBT) was assumed to be $90 \%$. The cooling capacity of the refrigerator was considered to be a typical value of $100 \mathrm{~W}$ (e.g., [8]). The classification of the heat amounts generated in a cryomodule is summarized in Fig. 3. Furthermore, $Q_{\text {ext }}$ could be estimated in the range of $10-30 \mathrm{~W}$ [9]; $Q_{\mathrm{RF}}$ and $Q_{\mathrm{b}}$ can be numerically evaluated by performing beam simulation and calculating the electromagnetic fields. On the other hand, $Q_{\text {emit }}$ can be effectively suppressed by appropriately designing the beam optics of LEBT (described in Chapter 2). Based on the aforementioned premise conditions, the feasibility of applying the

\begin{tabular}{|c|c|c|}
\hline$Q_{\mathrm{RF}}$ & Cause & Solution \\
\hline$Q_{b}$ & $\begin{array}{c}\text { RF Surface Resistance } \\
\text { (BCS Resistance + } \\
\text { Residual Resistance) }\end{array}$ & $\begin{array}{c}\text { High Q Cavity Performance } \\
\text { by Nb } 3 \text { Sn or N-doping }\end{array}$ \\
\hline$Q_{\text {ext }}$ & $\begin{array}{c}\text { Beam Collision } \\
\text { (to Inner Wall of RFQ) }\end{array}$ & $\begin{array}{c}\text { Optimization } \\
\text { of Design of RFQ }\end{array}$ \\
\hline to Cryo-Module from the outside & $\begin{array}{c}\text { Heat Penetration } \\
\text { Performance of Cryo-Module }\end{array}$ \\
\hline$Q_{\text {emit }}$ & $\begin{array}{c}\text { Beam Collisions } \\
\text { (to RFQ Entrance) }\end{array}$ & $\begin{array}{c}\text { Optimization } \\
\text { of Design of LEBT }\end{array}$ \\
\hline
\end{tabular}

FIG. 3. The classification of amount of heat generated in a cryomodule $[10,11]$.

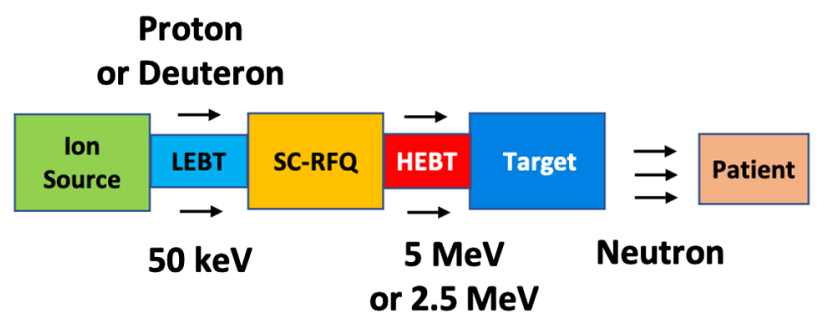

FIG. 4. Schematic of neutron source assumed in this study.
SRF cavity to the BNCT system was verified against the three criteria discussed earlier.

The procedure employed for the current study is summarized herein. First, we designed a LEBT that can suppress $Q_{\text {emit }}$ to 0 and designed a basic SC-RFQ structure with high beam-current transmission by using the RFQGen software. Subsequently, the beam simulation was performed to determine the beam trajectories in SC-RFQ, beam currents at the RFQ entrance, beam intensity at the RFQ exit, beam loss at each cell, detailed cell parameters of SC-RFQ, length of SC-RFQ, and the electromagnetic fields in SC-RFQ calculated by SUPERFISH [12]. Thus, $Q_{\mathrm{rf}}$ and $Q_{\mathrm{b}}$ can be numerically evaluated using these parameters. Moreover, $Q_{\mathrm{rf}}$ and $Q_{\mathrm{b}}$ vary with the peak surface E-field $E_{p}$. In this study, $Q_{\text {ext }}$ was considered to be $20 \mathrm{~W}$. The total amount of heat generated in a cryomodule $Q_{\text {tot }}$ was determined based on $E_{p}$, and $Q_{\text {tot }}$ was compared with the 100-W cooling capacity of the commercial helium refrigerator. Furthermore, the beam intensity at the RFQ exit was compared with the neutron production required for the medical treatment using BNCT. In addition, the length of the SC-RFQ was compared to other RFQs. Thereafter, the ac power consumption for SC-BNCT was evaluated based on the beam loading and the refrigerator operational power. In this study, the duration of the medical treatment using BNCT $T$ was set at $10 \mathrm{~h}$ per day. The beam loading was estimated by the beam intensity and efficiency of the $\mathrm{rf}$ source $\varepsilon_{\mathrm{rf}}$, and the $\varepsilon_{\mathrm{rf}}$ was set at 0.5. Moreover, the refrigerator power was estimated by considering the obtained amount of heat with the heat-removal efficiency of the refrigerator. In this study, the heat-removal efficiencies for $Q_{\mathrm{rf}}$ and $Q_{\mathrm{b}}$ were assumed to be $0.6 \mathrm{~kW} / \mathrm{W}$, whereas that for $Q_{\text {ext }}$ was chosen to be $1 \mathrm{~kW} / \mathrm{W}$. The ac power consumption of the SC-BNCT thus obtained was compared to that of the conventional BNCT.

The remainder of this paper is organized as follows. In Sec. II, we describe the designs of LEBT and the SC-RFQ, the premise conditions of beam simulation, and the method to evaluate the amount of heat. In Sec. III, the $Q_{\text {tot }}$ and RFQ length were evaluated as a function of $E_{p}$, and the ac power consumption was compared between the conventional and superconducting BNCT systems. The results of the feasibility study are discussed in Sec. IV. Finally, the overall results obtained from this study and the inferences are presented in Sec. V, concluding the feasibility of applying the superconducting $\mathrm{rf} \mathrm{Nb}$-cavity to the accelerator-based neutron source for BNCT.

\section{DESIGN OF AN ACCELERATOR FOR SC-BNCT SYSTEM}

In this chapter, we will explain the design of an accelerator for SC-BNCT systems, such as the specification of the ion source for deuteron/proton beams, the design of LEBT, an exemplary result of the deuteron/proton beam simulation, and the methods to evaluate $Q_{\mathrm{b}}$ and $Q_{\mathrm{rf}}$. 
TABLE II. Ion Source used in this study.

\begin{tabular}{lcc}
\hline \hline Parameter & Proton & Deuteron \\
\hline Energy $[\mathrm{keV}]$ & 50 & 50 \\
Current $[\mathrm{mA}]$ & 30 & 30 \\
Distribution & Water bag & Water bag \\
Transverse Emittance $[\mathrm{cm} \mathrm{mrad}]$ & 0.02 & 0.02 \\
Twiss parameters $(\alpha, \beta[\mathrm{cm} / \mathrm{mrad}])$ & $(1.02,3.10)$ & $(1.23,4.53)$ \\
\hline \hline
\end{tabular}

\section{A. Profile of ion source for SC-BNCT System}

The parameters specifying the ion source for deuteron/ proton beam are listed in Table II.

\section{B. Design of LEBT for SC-BNCT system}

The existing LEBT beam optics comprise an ion source, a RFQ, and two solenoid lenses. The trajectories of the beams can be focused on the RFQ entrance position by adjusting the strength of the magnetic fields, and its phase space can be matched to the RFQ acceptance. Although such LEBT structures are regarded as standard, they cannot be applied to SC-BNCT owing to the following reasons: (i) the beams emitted from an ion source generally contain a considerable \% of molecular ion beams. The RFQ is not designed to capture the charged particles that do not have the mass ratio of the target ion. Therefore, such molecular ion beams are rapidly lost at the RFQ entrance position, which causes heat amounts as $Q_{\text {emit }}$ (see Fig. 3). (ii) The beam emitted from the ion source generally contains a considerable $\%$ of poor emittance beams, which belong to the phase space far from RFQ acceptance. Thus, they are rapidly lost at the RFQ entrance position as well, which generates heat amounts of $Q_{\text {emit }}$. Hence, a considerable $\%$ of proton beams are lost at the entrance position of the $\mathrm{J}$ PARC RFQ [13]. For instance, a loss of 5\% for an incident beam of $(30 \mathrm{~mA}, 50 \mathrm{keV})$ results in a $Q_{\text {emit }}$ of $75 \mathrm{~W}$, which is highly significant in comparison to the $100-\mathrm{W}$ cooling capacity of the helium refrigerator. Thus, the beam optics of LEBT for SC-RFQ require the capability of perfectly separating the molecular ion and poor emittance beams.

In this study, we designed the beam optics of LEBT for SC-BNCT such that it eliminates the molecular ions and poor emittance beams. The beam optics of LEBT adopted in this study are summarized in Tables III and IV, which selected as beam behind ion source and that at RFQ entrance position are matched in RFQGen simulation software. The design concept is described below. The beam optics comprised a bending magnet, two normalconducting solenoids, and several orifices. First, the beams were narrowed with the orifice behind the ion source and guided toward the bending magnet for separating the trajectories between the target ion (proton or deuteron) and other molecular ions. The strength of each solenoid coil
TABLE III. LEBT design for proton acceleration. The charge neutralization effect was assumed to be $90 \%$ during LEBT transportation. The symbols $\theta$ and $\rho$ represent the bend angle and curvature radius for the bending magnet, respectively.

\begin{tabular}{ll}
\hline \hline Element & \multicolumn{1}{c}{ Specification } \\
\hline Drift & Length $1 \mathrm{~cm}$ \\
Orifice & Hole Radius $0.16 \mathrm{~cm}$ \\
Drift & Length $4.5 \mathrm{~cm}$ \\
Bending magnet & $\theta 15^{\circ}, \rho 3.23 \mathrm{~cm}$ \\
Drift & Length $14 \mathrm{~cm}$ \\
Solenoid & B-Strength $0.95 \mathrm{~T}$, Width $5 \mathrm{~cm}$ \\
Orifice & Hole radius $1.05 \mathrm{~cm}$ \\
Drift & Length $14 \mathrm{~cm}$ \\
Orifice & Hole radius $0.2 \mathrm{~cm}$ \\
Drift & Length $1 \mathrm{~cm}$ \\
Orifice & Hole radius $0.2 \mathrm{~cm}$ \\
Drift & Length $1 \mathrm{~cm}$ \\
Orifice & Hole radius $0.2 \mathrm{~cm}$ \\
Drift & Length $13 \mathrm{~cm}$ \\
Orifice & Hole radius $0.9 \mathrm{~cm}$ \\
Drift & Length $5 \mathrm{~cm}$ \\
Solenoid & B-strength $0.994 \mathrm{~T}$, Width $5 \mathrm{~cm}$ \\
Drift & Length $12 \mathrm{~cm}$ \\
\hline \hline
\end{tabular}

was adjusted to focus the target ion beam orbit at the midpoint of the two solenoid coils, and numerous orifices were installed near the focusing point. As the phase space ellipse of the beams rotated near the focusing point, the beams were reformed to correspond to the hall sizes of the orifices. Therefore, only the good emittance beams could pass through the orifices around the midpoint of the two solenoids, and consequently reached the RFQ entrance

TABLE IV. LEBT design for deuteron acceleration. The charge neutralization effect was assumed to be $90 \%$ during LEBT transportation. $\theta$ and $\rho$ represent the bend angle and curvature radius for the bending magnet, respectively.

\begin{tabular}{ll}
\hline \hline Element & \multicolumn{1}{c}{ Specification } \\
\hline Drift & Length $2 \mathrm{~cm}$ \\
Orifice & $3 \mathrm{~cm}$ \\
Drift & Length $3 \mathrm{~cm}$ \\
Bending magnet & $\theta 15^{\circ}, \rho 4.57 \mathrm{~cm}$ \\
Drift & Length $23 \mathrm{~cm}$ \\
Solenoid & B-strength $1 \mathrm{~T}$, Width $5 \mathrm{~cm}$ \\
Drift & Length $35 \mathrm{~cm}$ \\
Orifice & Hole radius $0.3 \mathrm{~cm}$ \\
Drift & Length $1 \mathrm{~cm}$ \\
Orifice & Hole radius $0.3 \mathrm{~cm}$ \\
Drift & Length $1 \mathrm{~cm}$ \\
Orifice & Hole radius $0.3 \mathrm{~cm}$ \\
Drift & Length $23 \mathrm{~cm}$ \\
Orifice & Hole radius $2 \mathrm{~cm}$ \\
Solenoid & B-strength $1.048 \mathrm{~T}$, Width $5 \mathrm{~cm}$ \\
Drift & Length $20.5 \mathrm{~cm}$ \\
\hline \hline
\end{tabular}




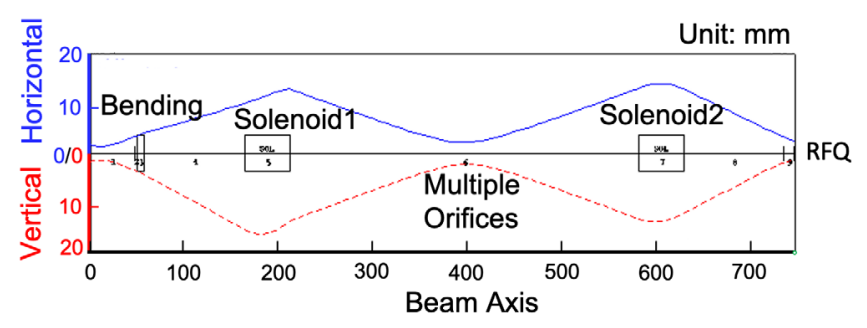

FIG. 5. Beam envelope for proton in LEBT.

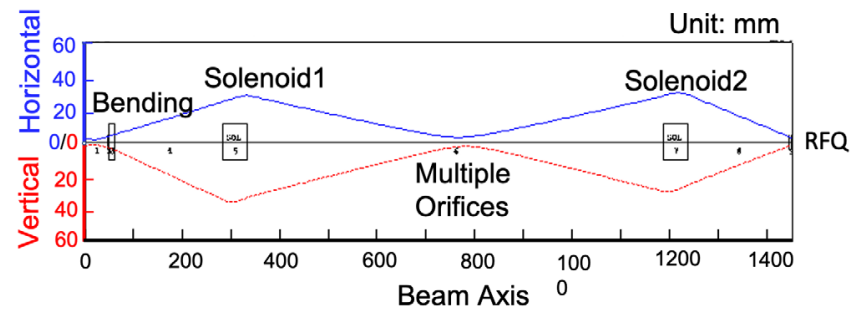

FIG. 6. Beam envelope for deuteron in LEBT.

position. Furthermore, the RFQGen simulation evaluated the beam current transmission ratio of the LEBT in Tables III and IV as $76.4 \%$ (for proton) and $66.9 \%$ (for deuteron).

For reference, Figs. 5 and 6 show the time evolution of the beam envelopes of the proton beam and deuteron beam in the LEBT of Tables III and IV evaluated by Trace-2D software. The horizontal axis represents the positions and layouts about the optics elements of which the LEBT consists, whereas the vertical axis represents the absolute values of the beam spread in the horizontal (x) and vertical (y) directions, respectively. The red and blue colors denote the beam spread in the $\mathrm{x}$ and $\mathrm{y}$ directions, respectively. As already mentioned, the charge neutralization effect during traveling in the LEBT was assumed to be $90 \%$. It should be noted that the beam envelope depicted in Figs. 5 and 6 do not contain the effect of orifices because Trace-2D does not have the function to represent such an aperture. It should also be noted that the length of LEBT and the replacements of solenoid and bending magnets are not exactly same as those summarized in Tables III and IV. This is probably due to a subtle difference in the effect of the leakage magnetic field at edge point and the criterion of the space charge effect defined between RFQGen and Trace-2D software.

Fig. 7 and Fig. 8 display scatter plots of $x-x^{\prime}$ and $y-y$ ' distributions at the RFQ entrance position obtained from LEBT beam simulation, respectively. The top and bottom rows represent the results for $0.02 \mathrm{~cm} \mathrm{mrad}$ and $0.2 \mathrm{~cm}$ mrad, respectively, which shows that the phase space distribution at the RFQ entrance position remained almost the same even when the emittance deteriorated by 10 times in comparison to the reference value of $0.02 \mathrm{~cm} \mathrm{mrad}$. Although there was a slight difference between the results of $0.02 \mathrm{~cm} \mathrm{mrad}$ and $0.2 \mathrm{~cm} \mathrm{mrad}$, we confirmed that the
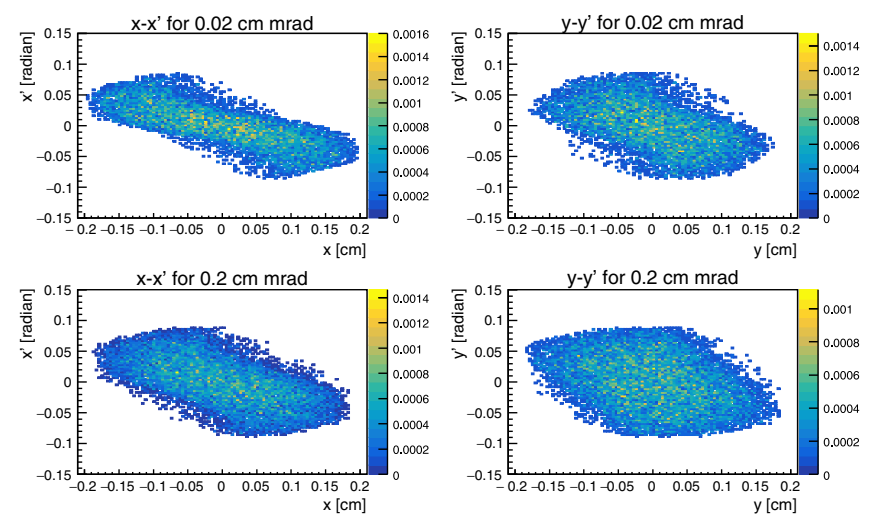

FIG. 7. Scatter plot for the phase space distribution of proton beams at the RFQ entrance. The top two figures correspond to the simulation result with normalized emittance of $0.02 \mathrm{~cm} \mathrm{mrad}$, whereas the bottom two figures correspond to that with $0.2 \mathrm{~cm}$ mrad. These scatter plots are created by the number of MC events of 10,000 of LEBT beam simulation conducted with RFQGen software and the integrated numbers of them are normalized to be 1 . These histograms are divided with $100 \times 100$ bins.

RFQ beam simulation results indicated that the beam transmission rate did not deteriorate in both cases of Figs. 7 and 8. The RFQ simulation was performed with the LEBT.

\section{Design of the RFQ for the SC-BNCT system}

In this study, the structure of the RFQ was determined by RFQGen. The input parameters are summarized in Table V. The cell parameters of the RFQ are summarized in Tables VI-VIII. The strength of the peak surface electric field and the minimum aperture tended to be slightly lower than the input values.
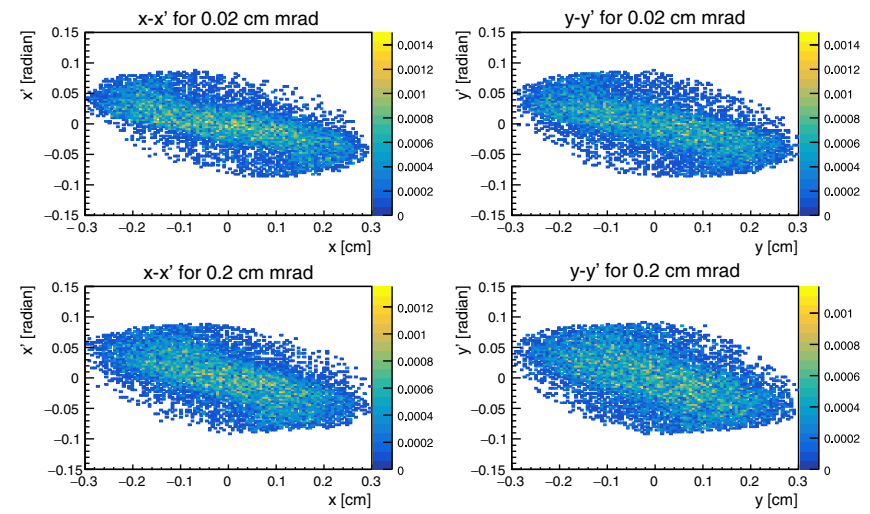

FIG. 8. Scatter plot for the phase space distribution of deuteron beams at the RFQ entrance. The top two figures correspond to the simulation result with normalized emittance of $0.02 \mathrm{~cm} \mathrm{mrad}$, whereas the bottom two figures correspond to that with $0.2 \mathrm{~cm}$ mrad. These scatter plots are created by the number of MC events of 10,000 of LEBT beam simulation conducted with RFQGen software and the integrated numbers of them are normalized to be 1 . These histograms are divided with $100 \times 100$ bins. 
TABLE V. Input parameter list for RFQGen for proton/deuteron acceleration simulation. Actually, the peak surface E-field $E_{p}$ and the minimum aperture $a_{0}$ determined from the RFQGen software were slightly lower than the values of the input parameters.

\begin{tabular}{lc}
\hline \hline Input & Details \\
\hline Beam energy [MeV] (for proton) & 2.5 or 5 \\
Beam energy [MeV] (for deuteron) & 5 \\
Synchronous phase [deg] & -35 \\
The number of RM cells & 4 \\
Incident energy [keV] & 50 \\
Energy at shaper exit [keV] & 55 \\
$a_{0}[\mathrm{~cm}]$ (for proton) & 0.32 \\
Resonant frequency [MHz] (for proton) & 325 \\
$E_{p}[\mathrm{MV} / \mathrm{m}]$ (for proton) & $40,45,50,55,60$ \\
$a_{0}[\mathrm{~cm}]$ (for deuteron) & 0.48 \\
Resonant frequency [MHz] (for deuteron) & 162.5 \\
$E_{p}[\mathrm{MV} / \mathrm{m}]$ (for deuteron) & $35,40,45,50,55$ \\
\hline \hline
\end{tabular}

For reference, the results of beam simulation of $2.5 \mathrm{MeV}$ proton are presented in Fig. 9. In addition, the phase space distribution, beam spread, synchronous phase spread, and energy spread at the RFQ exit are depicted in Fig. 10. Normalized beam emittance and Twiss parameters obtained from beam simulation of $2.5 \mathrm{MeV}$ proton, beam simulation of $5 \mathrm{MeV}$ proton, and beam simulation of $5 \mathrm{MeV}$ deuteron are summarized in Tables IX and X.
Figure 11 illustrates the plot of the percentage of beam current that can pass through the RFQ and reach the RFQ exit. The horizontal axis represents $E_{p}$ and the vertical axis accounts for the ratio of beam current at RFQ exit to that at RFQ entrance. In each case, a transmission ratio of $99.8 \%$ or more was achieved. These transmission rates should be referred as to "theoretical transmission" because the results obtained here do not contain effects of errors due to RFQ fabrication and alignment of the RFQ body. The beam currents at the RFQ exit assumed in this study were $22.9 \mathrm{~mA}$ (for proton) and $20.3 \mathrm{~mA}$ (for deuteron), which satisfied the beam intensity requirements of the BNCT presented in Table I.

\section{Method for evaluating rf energy loss}

A method to evaluate the dissipated power of the ohmic loss of SC-RFQ is described herein. Prior to performing the beam simulation of the RFQGen, the cell parameters of the RFQ structure were determined to create an input file for the electromagnetic code of SUPERFISH. Therefore, the dissipated power per unit length for the 2D geometry of a quadrant of the normal conducting RFQ, $W_{\mathrm{SF}}$, can be evaluated by electromagnetic calculation with SUPERFISH. Consequently, the dissipated power of the ohmic loss of the SC-RFQ $Q_{\mathrm{rf}}$ can be numerically evaluated as follows:

$$
Q_{\mathrm{rf}}=4 \frac{R_{\mathrm{Nb}}}{R_{\mathrm{Cu}}} W_{\mathrm{SF}} L_{\mathrm{RFQ}}
$$

TABLE VI. Cell parameters designed using RFQGen software with SC-RFQ for $2.5 \mathrm{MeV}$ proton beam acceleration.

\begin{tabular}{lccccc}
\hline \hline$E_{p}$ (input) [MV/m] & 40 & 45 & 50 & 55 & 60 \\
\hline Cell number & 431 & 362 & 312 & 271 & 240 \\
Focusing parameter $B$ & $0.01-5.56$ & $0.01-6.11$ & $0.01-6.67$ & $0.01-7.19$ & $0.01-7.70$ \\
Final modulation factor & 2.0420 & 2.0533 & 2.0621 & 2.0742 & 2.0816 \\
$a_{0}$ (minimum value) [mm] & 2.664 & 2.677 & 2.653 & 2.662 & 2.633 \\
$r_{0}$ (mean value) $[\mathrm{mm}]$ & 4.431 & 4.573 & 4.679 & 4.864 & 4.964 \\
$E_{p}$ (designed) $[\mathrm{MV} / \mathrm{m}]$ & 35.1 & 39.3 & 43.2 & 47.6 & 51.3 \\
Vane voltage $[\mathrm{kV}]$ & 111.5 & 127.2 & 141.1 & 159.6 & 172.6 \\
\hline \hline
\end{tabular}

TABLE VII. Cell parameters designed using RFQGen software with SC-RFQ for $5 \mathrm{MeV}$ proton beam acceleration.

\begin{tabular}{lccccc}
\hline \hline$E_{p}$ (input) $[\mathrm{MV} / \mathrm{m}]$ & 40 & 45 & 50 & 55 & 60 \\
\hline Cell number & 491 & 414 & 357 & 312 & 276 \\
Focusing parameter $B$ & $0.01-5.56$ & $0.01-6.11$ & $0.01-6.67$ & $0.01-7.19$ & $0.01-7.70$ \\
Final modulation factor & 2.0343 & 2.0353 & 2.0361 & 2.0368 & 2.0375 \\
$a_{0}$ (minimum value) $[\mathrm{mm}]$ & 2.656 & 2.676 & 2.673 & 2.660 & 2.658 \\
$r_{0}$ (mean value) $[\mathrm{mm}]$ & 4.407 & 4.545 & 4.682 & 4.814 & 4.964 \\
$E_{p}$ (design) $[\mathrm{MV} / \mathrm{m}]$ & 35.8 & 40.1 & 44.5 & 48.6 & 52.9 \\
Vane voltage $[\mathrm{kV}]$ & 111.5 & 127.4 & 143.8 & 159.8 & 177.0 \\
\hline \hline
\end{tabular}


TABLE VIII. Cell parameters designed using RFQGen software with SC-RFQ for $5 \mathrm{MeV}$ deuteron beam acceleration.

\begin{tabular}{lccccc}
\hline \hline$E_{p}$ (input) $[\mathrm{MV} / \mathrm{m}]$ & 35 & 40 & 45 & 50 & 55 \\
\hline Cell number & 371 & 307 & 260 & 225 & 197 \\
Focusing parameter $B$ & $0.01-6.33$ & $0.01-7.04$ & $0.01-7.74$ & $0.01-8.42$ & $0.01-9.07$ \\
Final modulation factor & 2.0363 & 2.0374 & 2.0384 & 2.0393 & 2.0403 \\
$a_{0}$ (minimum value) $[\mathrm{mm}]$ & 4.003 & 3.984 & 3.966 & 3.957 & 3.959 \\
$r_{0}$ (mean value) $[\mathrm{mm}]$ & 6.811 & 7.075 & 7.360 & 7.647 & 7.977 \\
$E_{p}$ (design) $[\mathrm{MV} / \mathrm{m}]$ & 30.75 & 34.90 & 39.07 & 43.24 & 47.53 \\
Vane voltage $[\mathrm{kV}]$ & 145.6 & 168.7 & 192.9 & 217.5 & 244.3 \\
\hline \hline
\end{tabular}

where $R_{\mathrm{Nb}}$ denotes the rf surface resistance of the SC-RFQ made of $\mathrm{Nb}, R_{\mathrm{Cu}}$ denotes the rf surface resistance of NCRFQ coated with thick copper films, $L_{\mathrm{RFQ}}$ denotes the length of the SC-RFQ, and a multiple factor of 4 is necessary for evaluating the ohmic loss generated from the total RFQ geometry.

$R_{\mathrm{Nb}}$ comprises two terms: BCS resistance $R_{\mathrm{BCS}}$ and residual resistance $R_{\text {res }}$ [see Eq. (A4)]. In this study, we adopted $R_{\mathrm{BCS}}$ as the semiempirical formula of $\mathrm{BCS}$ resistance of bulk $\mathrm{Nb}$ of Eq. (A5). On the contrary, we adopted $43 \mathrm{n} \Omega$ as $R_{\text {res }}$ the residual resistance of $350 \mathrm{MHz}$ CH-DTL whose TE21 resonant mode was the same as that of the RFQ [14].

\section{E. Method for evaluating the heat amount due to beam collisions}

The following equation was used to evaluate the amount of heat owing to beam collisions $Q_{\mathrm{b}}$ :

$$
Q_{\mathrm{b}}=\sum I \cdot W_{\mathrm{i}} \frac{\Delta N_{\mathrm{i}}}{N}
$$

where $\mathrm{i}$ represents the index of ith cells of RFQ, $W_{\mathrm{i}}$ represents the energy that MC particles lose at the ith cell, $I$

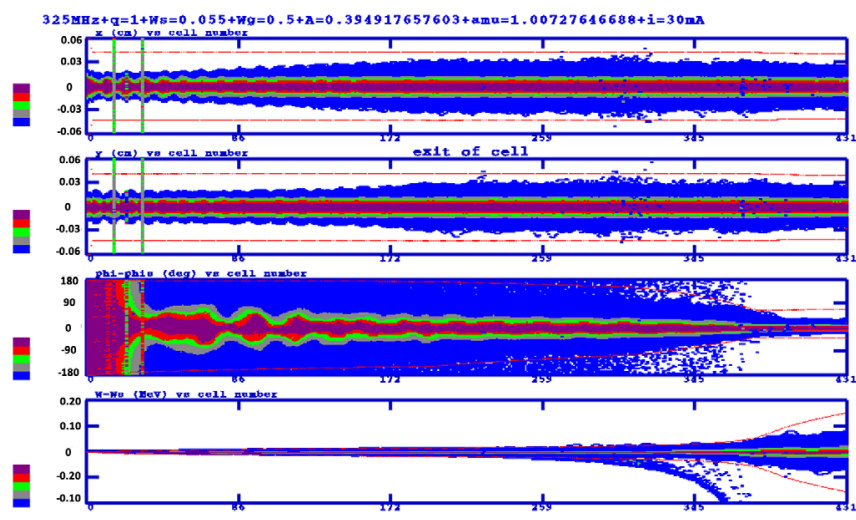

FIG. 9. RFQGen beam dynamics simulation for $2.5 \mathrm{MeV}$ proton. The horizontal axis represents the number of cells in the RFQ. Top: $x$ vs RFQ cells. (second from the top) y vs RFQ cells. (third from the top) the spread of synchronous phase vs RFQ cells. Bottom: energy spread vs RFQ cells. corresponds to the beam current emitted from the ion source, $\mathrm{N}$ is the total number of MC particles, and $\Delta N_{\mathrm{i}}$ represents the number of $\mathrm{MC}$ particles lost at the ith cell.

In general, the deposit energy of the lost particles in each cell $Q_{\mathrm{b}}$ suffers from an error caused by the statistical uncertainty of MC particles. In this study, we evaluated the error of $Q_{\mathrm{b}}$ by calculating the variance $V$ of the binomial distribution defined from the number of MC particles entering each cell and that are lost at each cell; $\sqrt{V}$ was considered as the statistical fluctuation of MC particles lost at the cell. On the contrary, the beam current entering the RFQ entrance suffers from statistical uncertainty, because the original beam current is partially lost at the orifices installed in the LEBT. Hereafter, $N$ and $N^{\prime}$ are defined as the number of $\mathrm{MC}$ particles used in this simulation and that through LEBT, respectively. If the beam current is partially lost in LEBT, $I$ and $N$ of Eq. (2) should be changed as $I \rightarrow \frac{N^{\prime}}{N} I$ and $N \rightarrow N^{\prime}$. Each term suffers from the statistical uncertainty of $N^{\prime}$. Thus, we added such statistical fluctuations to the uncertainty of $Q_{\mathrm{b}}$.
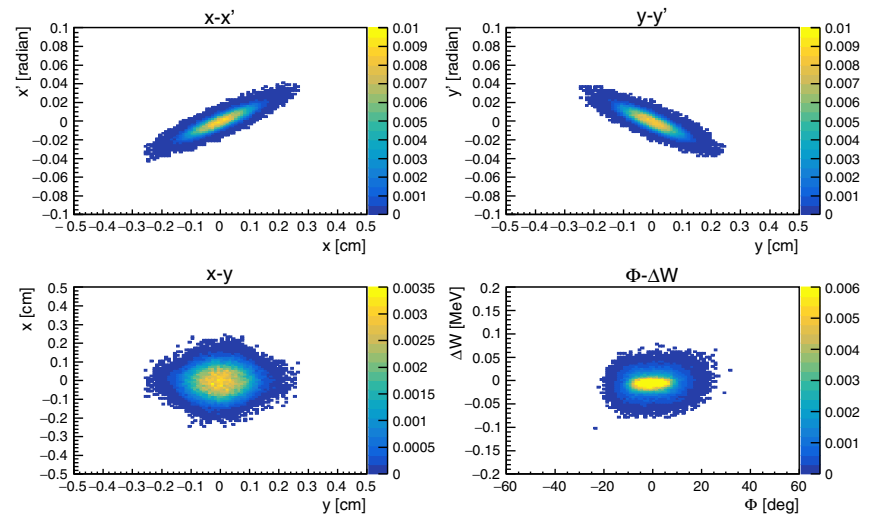

FIG. 10. Beam emittance, beam spread, phase spread, and energy spread for $2.5-\mathrm{MeV}$ simulation. The top-left and top-right correspond to $\mathrm{x}-\mathrm{x}$ ' and $\mathrm{y}-\mathrm{y}$ ' (cm-mrad), respectively. The bottomleft denotes the beam spread $(\mathrm{cm})$. The bottom-right denotes energy spread-phase spread. Theses scatter plots are created by the number of $\mathrm{MC}$ events of beam simulation conducted with RFQGen software and the integrated numbers of them are normalized to be 1 . These histograms are divided with $100 \times 100$ bins. 
TABLE IX. Twiss parameters and RMS emittance at RFQ entrance position determined with RFQGen software. Each cell contains a data represented as A/B. A denote the obtained result for proton $2.5 \mathrm{MeV}$ simulation, and $\mathrm{B}$ denote the obtained result for proton $5 \mathrm{MeV}$ simulation.

\begin{tabular}{lccccc}
\hline \hline$E_{p}$ (input) [MV/m] & 40 & 45 & 50 & 55 & 60 \\
\hline$\alpha_{x}$ [a.u.] & $-1.85 /-1.65$ & $1.527 / 1.55$ & $-1.818 /-1.589$ & $-1.718 / 1.502$ & $-1.756 / 1.599$ \\
$\beta_{x}$ [cm mrad] & $0.200 / 0.170$ & $0.146 / 0.150$ & $0.159 / 0.139$ & $0.143 / 0.122$ & $0.136 / 0.122$ \\
$\varepsilon_{x}$ [cm/mrad] & $0.0236 / 0.0237$ & $0.0259 / 0.0257$ & $0.0260 / 0.0258$ & $0.0256 / 0.0256$ & $0.0276 / 0.0268$ \\
$\alpha_{y}$ [a.u.] & $1.600 / 1.751$ & $-1.885 /-1.544$ & $1.465 /-1.506$ & $1.483 /-1.550$ & $1.473 /-1.498$ \\
$\beta_{y}$ [cm/mrad] & $0.168 / 0.172$ & $0.182 / 0.152$ & $0.130 / 0.135$ & $0.116 / 0.127$ & $0.107 / 0.117$ \\
$\varepsilon_{y}$ [cm mrad] & $0.0235 / 0.0233$ & $0.0246 / 0.0247$ & $0.0252 / 0.0251$ & $0.0251 / 0.0251$ & $0.0255 / 0.0256$ \\
$\alpha_{z}$ [a.u.] & $-0.103 /-0.141$ & $-0.151 /-0.151$ & $-0.153 /-0.125$ & $-0.245 /-0.049$ & $-0.319 / 0.022$ \\
$\beta_{z}$ [cm $\left./ \mathrm{mrad}\right]$ & $0.385 / 0.345$ & $0.343 / 0.288$ & $0.311 / 0.253$ & $0.291 / 0.211$ & $0.273 / 0.183$ \\
$\varepsilon_{z}$ [cm mrad] & $0.0322 / 0.0332$ & $0.0358 / 0.0377$ & $0.0428 / 0.0436$ & $0.0520 / 0.0533$ & $0.0556 / 0.0574$ \\
\hline \hline
\end{tabular}

TABLE X. Twiss parameters and RMS emittance at RFQ entrance position determined with RFQGen software obtained when deuteron $5 \mathrm{MeV}$ beam simulation is conducted.

\begin{tabular}{lccccc}
\hline \hline $\begin{array}{l}E_{p} \text { (input) } \\
{[\mathrm{MV} / \mathrm{m}]}\end{array}$ & 35 & 40 & 45 & 50 & 55 \\
\hline$\alpha_{x}$ [a.u. & -1.44689 & -1.4914 & 1.484 & -1.376 & -1.543 \\
$\beta_{x}[\mathrm{~cm} \mathrm{mrad}]$ & 0.278 & 0.250 & 0.242 & 0.191 & 0.191 \\
$\varepsilon_{x}[\mathrm{~cm} / \mathrm{mrad}]$ & 0.0314 & 0.0338 & 0.0366 & 0.0392 & 0.0417 \\
$\alpha_{y}[\mathrm{a} . \mathrm{u}]$. & 1.558 & 1.564 & -1.484 & 1.511 & 1.670 \\
$\beta_{y}[\mathrm{~cm} / \mathrm{mrad}]$ & 0.306 & 0.274 & 0.218 & 0.223 & 0.214 \\
$\varepsilon_{y}[\mathrm{~cm} \mathrm{mrad}]$ & 0.0314 & 0.0347 & 0.0366 & 0.0395 & 0.0433 \\
$\alpha_{z}[\mathrm{a} . \mathrm{u}]$. & -0.13596 & -0.07802 & -0.12864 & -0.15963 & -0.2601 \\
$\beta_{z}[\mathrm{~cm} / \mathrm{mrad}]$ & 0.244 & 0.207 & 0.169 & 0.151 & 0.159 \\
$\varepsilon_{z}[\mathrm{~cm} \mathrm{mrad}]$ & 0.0451 & 0.0524 & 0.059 & 0.0657 & 0.0736 \\
\hline \hline
\end{tabular}

\section{F. Method for evaluating ac power consumption}

This subsection introduces the mathematical equations to evaluate the ac power consumption of the BNCT system with a linear-accelerator-based neutron source using the NRF cavity $W_{\mathrm{n}}$ and that using the SRF cavity $W_{\mathrm{s}}$, respectively. Hereafter, we neglect the ac power consumption necessary for the working of a water-cooling system to simplify the following discussion. Furthermore, the beam operation period for one day was defined as $T$, an electric power efficiency for the rf source as $\frac{1}{\varepsilon_{\mathrm{rt}}}$, and the total surface heat amounts as $Q_{\text {surf }}=Q_{\mathrm{rf}}+Q_{\mathrm{b}}$.

First, a linear-accelerator-based neutron source using the NRF cavity generates ac power consumption to drive the rf source for particle acceleration only during the operation of BNCT. Thus, we defined the input power into the NRF cavity as $P_{n \text {,in }}$ for particle acceleration. Therefore, the ac power consumption for driving the BNCT system in this case for one day can be expressed as follows:

$$
W_{\mathrm{n}}=\frac{P_{n, \mathrm{in}}}{\varepsilon_{\mathrm{rf}}} T
$$

Thereafter, a linear-accelerator-based neutron source using the SRF cavity generated two types of ac power consumption: (1) an input power to drive the rf source for particle acceleration, which is referred to as $P_{s, \text { in }}$. (2) The heat-removal power of refrigerator for the heat generated in a cryomodule such as $Q_{\text {surf }}$ and $Q_{\text {ext }}$ (see Fig. 3). The removal of $Q_{\text {surf }}$ is needed only during the operation for the medical treatment of BNCT. On the contrary, the removal of $Q_{\text {ext }}$ is required for all the days during the period. In order to express that the cooling efficiency of a refrigerator increases as the amount of heat removed increases, we introduced two types of refrigerator efficiencies: $\varepsilon_{\text {surf }}$ and $\varepsilon_{\text {ext }}$, where $\varepsilon_{\text {surf }}$ denotes the removal of $Q_{\text {surf }}$ and $\varepsilon_{\text {ext }}$ represents that for $Q_{\text {ext }}$. From these discussions, the ac power consumption for driving the SC-BNCT system $W_{\mathrm{s}}$ can be expressed as follows:

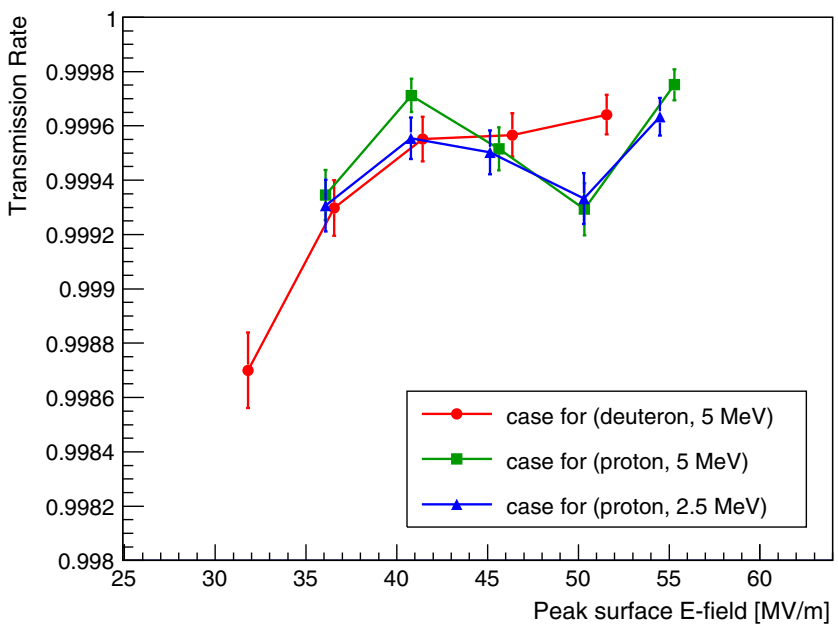

FIG. 11. Transmission rate through RFQ compared to the beam intensity at the RFQ entrance as a function of peak surface Efield. The vertical and horizontal axes represent the transmission rate and the peak surface E-field, respectively. The red circles denote the result of 5-MeV deuteron, triangles denote the result of $5-\mathrm{MeV}$ proton, and the squares denote the result of $5-\mathrm{MeV}$ deuteron. 


$$
W_{\mathrm{s}}=\frac{P_{n, \mathrm{in}}}{\varepsilon_{\mathrm{rf}}} T+Q_{\text {surf }} \varepsilon_{\mathrm{surf}} T+Q_{\mathrm{ext}} \varepsilon_{\mathrm{ext}} 24
$$

\section{EVALUATION OF THE SUPERCONDUCTING BNCT SYSTEM}

\section{A. Evaluation of heat loading}

A comparison plot of $Q_{\mathrm{b}}$ and $Q_{\mathrm{rf}}$ evaluated by the simulation between various $E_{p}$ values is depicted in Figs. 12 and 13. The horizontal and vertical axes represent $E_{p}$ and surface heat amounts of $Q_{\mathrm{b}} / Q_{\mathrm{rf}}$, respectively. Red curves denote the result of $5-\mathrm{MeV}$ deuteron, green curves denote the result of $5-\mathrm{MeV}$ proton, and the blue curves denote the result of 2.5-MeV proton. From Fig. 12, the $Q_{\mathrm{b}}$ for $2.5-\mathrm{MeV}$ proton amounted to $6-9 \mathrm{~W}$, that for $5-\mathrm{MeV}$ proton amounted to $3-11 \mathrm{~W}$, and that for $5-\mathrm{MeV}$ deuteron amounted to 5-14 W, respectively. Moreover, $Q_{\mathrm{rf}}$ in Fig. 13 tends to slightly increase with $E_{p}$. In addition, $Q_{\mathrm{rf}}$ for protons of $2.5 \mathrm{MeV}$ amounted to 7-11 $\mathrm{W}$, that for protons of $5 \mathrm{MeV}$ amounted to $13-18 \mathrm{~W}$, and that for deuterons amounted to 8-12 $\mathrm{W}$, respectively.

Using the simulation results of Figs. 12 and 13, we evaluated the total amount of heat generated in a cryomodule $Q_{\text {tot }}$ as a function of $E_{p}$. As discussed earlier, $Q_{\text {ext }}$ was considered $20 \mathrm{~W}$. Generally, the particle acceleration cannot be maintained using the SRF cavity if $Q_{\text {tot }}$ is greater than the cooling capacity of a commercially available $4.2 \mathrm{~K}$ helium refrigerator, but $Q_{\text {tot }}$ calculated by Figs. 12 and 13 were less than $100 \mathrm{~W}$ under any case considering the error bars. Thus, $Q_{\text {tot }}$ can be sufficiently suppressed within the cooling capacity of the refrigerator considered in this study,

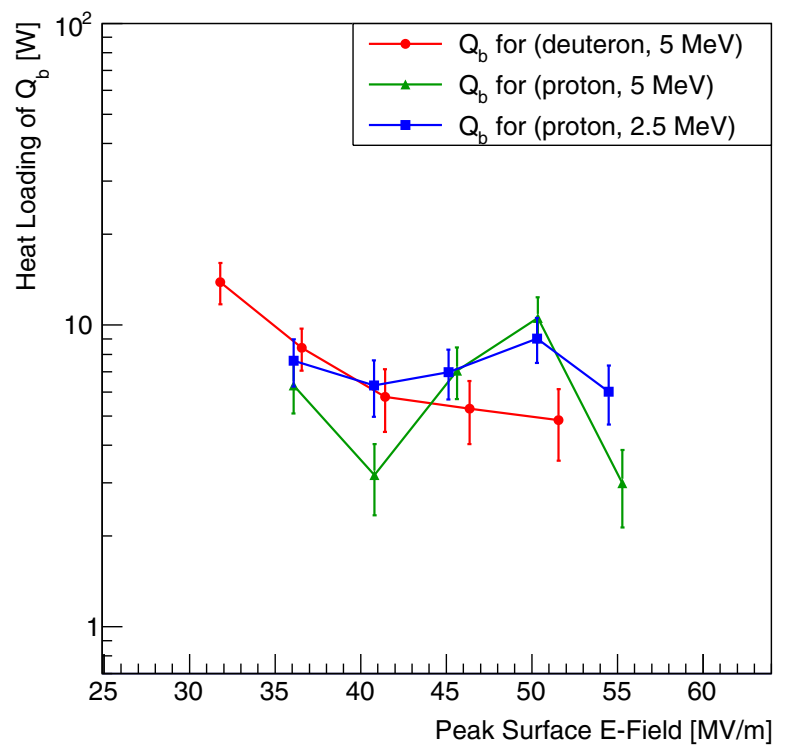

FIG. 12. Heat loading owing to beam loss as a function of peak surface-E field. $Q_{\mathrm{b}}$ shown in vertical axis correspond to the heat loading due to "theoretical transmission" shown in Fig. 11.

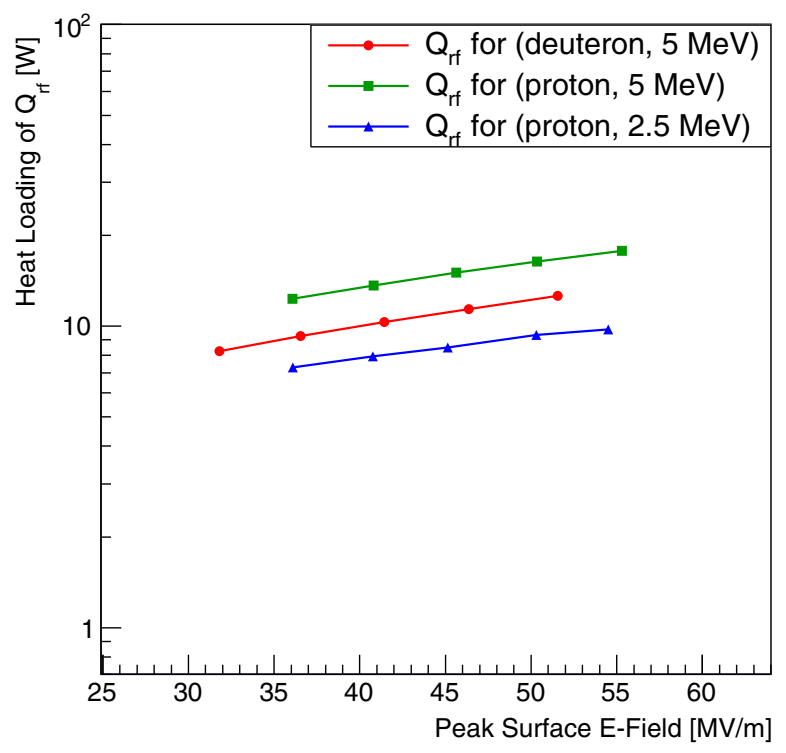

FIG. 13. Heat loading owing to rf resistance as a function of peak surface-E field.

i.e., $E_{p}>35 \mathrm{MV} / \mathrm{m}$ (for proton) and $E_{p}>30 \mathrm{MV} / \mathrm{m}$ (for deuteron), which guarantees the feasibility of SC-BNCT from the perspective of refrigerator loading.

\section{B. Evaluation of the RFQ length}

The RFQ length considered in this study design is shown in Fig. 14. In addition, the RFQ lengths of IFMIF RFQ, SNS RFQ, and J-PARC RFQ were also superimposed for comparison with the existing normal-conducting RFQs all over the world $[13,15,16]$. Figure 14 depicts that the length

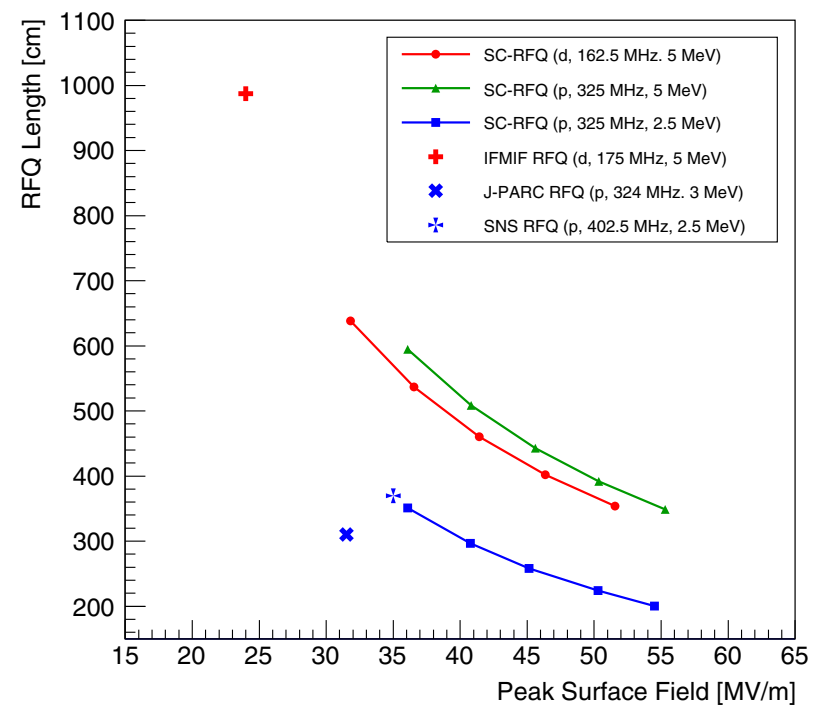

FIG. 14. RFQ length of SC cavities as a function of peak surface E-field. The vertical and horizontal axes represent the RFQ length and peak surface E-field, respectively. Moreover, the RFQ lengths of the NC cavities are plotted for reference. 
of the SC-RFQ decreases as the $E_{p}$ increases. The RFQ lengths for $2.5-\mathrm{MeV}$ proton acceleration are comparable with that of the SNS RFQ and J-PARC RFQ at $35-40 \mathrm{MV} / \mathrm{m}$, and it decreased at $E_{p}>40 \mathrm{MV} / \mathrm{m}$. Eventually, the RFQ length for a proton of $2.5 \mathrm{MeV}$ with $E_{p}=55 \mathrm{MV} / \mathrm{m}$ is reduced to half of the existing RFQs. In addition, the RFQ length for $5 \mathrm{MeV}$ deuteron at $E_{p}=30 \mathrm{MV} / \mathrm{m}$ acceleration has already been shortened to half of that of IFMIF RFQ, and it is almost one-third of the length of IFMIF RFQ at $E_{p}=50 \mathrm{MV} / \mathrm{m}$. This analysis indicates that a more compact SC-BNCT system can be designed if $E_{p}>30 \mathrm{MV} / \mathrm{m}$ for the deuteron accelerator and $E_{p}>40 \mathrm{MV} / \mathrm{m}$ for the proton accelerator, as compared to the existing BNCT systems.

\section{Evaluation of ac power consumption}

In this section, a numerical comparison of ac power consumption between the BNCT system with NC-RFQ and that of the BNCT system with SC-RFQ has been presented for a usage period of one day. As discussed earlier, we did not consider the ac power consumption required for the operation of a water-cooling system for simplicity. Moreover, Eq. (3) and Eq. (4) were used to evaluate the amount of heat. In addition, we selected the variables as the following set: the treatment time per day of BNCT $T=10 \mathrm{~h}$, electric power efficiency of the rf source $\varepsilon_{\mathrm{rf}}=0.5$, heat amounts penetrating into the cryomodule from the outside $Q_{\text {ext }} 20 \mathrm{~W}$, an electric power efficiency of a refrigerator to remove surface heat amounts $\varepsilon_{\text {surf }}$ $0.6 \mathrm{~kW} / \mathrm{W}$, and an electric power efficiency of the refrigerator to remove the heat penetrating into the cryomodule $\varepsilon_{\mathrm{b}} 1 \mathrm{~kW} / \mathrm{W}$. Furthermore, two case studies as demonstrative examples have been presented herein.

\section{Comparison of BNCT system of National Cancer Center at Japan}

The ac power consumption of the BNCT system of the National Cancer Research Center (NCC), Japan [1] was compared to that of a SC-BNCT system. The BNCT system operated at NCC has adopted an accelerator-based neutron source that utilizes a $20 \mathrm{~mA} \mathrm{CW}$ and $2.5 \mathrm{MeV}$ proton beams as incidents to the lithium target for neutron production and used $330 \mathrm{~kW} \mathrm{CW}$ klystron as the rf source. Thus, the ac power consumption of the conventional BNCT system operated at NCC is estimated to be $W_{\mathrm{n}}=330 \times 2 \times 10=6.6$ MWh. Similarly, we estimated the ac power consumption of a corresponding SC-BNCT system as shown below. The obtained result for the $2.5 \mathrm{MeV}$ proton beam simulation was used for comparison. The beam current for SC-BNCT was assumed to be $20 \mathrm{~mA}$, similar to that for the NCC. For a conservative evaluation, the peak surface E-field was set as $E_{p}=40 \mathrm{MV} / \mathrm{m}$. In addition, the output power of the rf source was $50 \mathrm{~kW}$. Therefore, the ac power consumption for the rf-powered
SC-BCNT system was evaluated as $P_{s, \text { in }} \varepsilon_{\mathrm{rf}} T=$ $50 \times 2 \times 10=1 \mathrm{MWh}$. Moreover, from Figs. 12 and 14, $Q_{\text {surf }}$ for $325 \mathrm{MHz}$ SC-RFQ can be evaluated as $14 \mathrm{~W}$ at $E_{p}=40 \mathrm{MV} / \mathrm{m}$ and the corresponding ac power consumption was estimated to be $Q_{\text {surf }} \varepsilon_{\text {surf }} T \sim 14 \times 0.6 \times 10=$ $84 \mathrm{kWh}$. In addition, from the premise condition of this study, the ac power consumption required for removing the heat penetrating the cryomodule from the outside was estimated as $Q_{\text {ext }} \varepsilon_{\text {ext }} 24=480 \mathrm{kWh}$. Thus, the ac power consumption of the SC-BNCT system was calculated at 1.56 MWh. In conclusion, the SC-BNCT system can reduce the ac power consumption to $W_{\mathrm{s}} / W_{\mathrm{n}}=0.236 \sim$, which is one-fourth in comparison to the conventional BNCT system operated at NCC.

\section{Comparison of BNCT system proposed at INFN-LNL}

The ac power consumption of the BNCT system proposed by INFN-LNL [17] was compared to that of the SC-BNCT system. First, the ac power consumption of the BNCT system proposed by INFN-LNL was evaluated. The INFN utilizes an accelerator-based neutron source in which $30 \mathrm{mACW}$ proton beams are accelerated and made incident onto beryllium to produce neutrons [17]. The estimated if power $P_{\mathrm{n}}=1 \mathrm{MW}$. Thus, the ac power consumption of the conventional BNCT system that will operate at INFN is estimated to be $W_{\mathrm{n}}=1 \times 10=10 \mathrm{MWh}$. Similarly, the ac power consumption of the corresponding SC-BNCT system was evaluated as follows. The results of beam simulations for the $5-\mathrm{MeV}$ proton and deuteron beams were further compared. Moreover, the beam currents at the RFQ exit were assumed to be $20 \mathrm{~mA}$ (for proton) $/ 15 \mathrm{~mA}$ (for deuteron), which were slightly lesser than the beam current estimated from the RFQGen simulation performed in this study. For a conservative evaluation, the peak surface E-field was set as $E_{p}=40 \mathrm{MV} / \mathrm{m}$ (for proton) and $30 \mathrm{MV} / \mathrm{m}$ (for deuteron). Moreover, the output power of the rf source was $100 \mathrm{~kW}$ (for proton)/75 $\mathrm{kW}$ (for deuteron), and the corresponding ac power consumption was evaluated as $2 \mathrm{MWh}$ (for proton)/ 1.5 MWh (for deuteron). In addition, from Figs. 12 and 13, $Q_{\text {surf }}$ for the $325-\mathrm{MHz}$ SC-RFQ at $E_{p}=40 \mathrm{MV} / \mathrm{m}$ and $162.5-\mathrm{MHz} \mathrm{SC}-\mathrm{RFQ}$ at $E_{p}=30 \mathrm{MV} / \mathrm{m}$ was evaluated as $17 \mathrm{~W}$ (for proton) and $22 \mathrm{~W}$ (for deuteron), and the corresponding ac power consumption were estimated as $102 \mathrm{kWh}$ (for proton) and $132 \mathrm{kWh}$ (for deuteron). Furthermore, the ac power consumption to remove the heat penetrating into a cryomodule from the outside as per the premise condition of this study was estimated as $Q_{\text {ext }} \varepsilon_{\text {ext }} 24=480 \mathrm{kWh}$. Thus, the ac power consumption of the SC-BNCT system was evaluated by adding each evaluated term and was obtained as $2.58 \mathrm{MWh}$ (for proton) and 2.11 MWh (for deuteron). In conclusion, the SC-BNCT system can reduce the ac power consumption to $W_{\mathrm{s}} / W_{\mathrm{n}}=$ 0.258 (for proton) and 0.211 (for deuteron) , which is $20 \%$ $25 \%$ of a conventional BNCT system. 


\section{DISCUSSION}

Several studies have been conducted on the design and fabrication of SC-RFQ. The Argonne National Laboratory (ANL, USA) designed and fabricated a prototype of SCRFQ of $200 \mathrm{MHz}$ in 1993, and they performed the LLRF test to measure two resonant frequencies of the quadrupole and dipole modes [18]. In addition, the Los Alamos National Laboratory (LANL, USA) designed and developed a prototype of SC-RFQ of $425 \mathrm{MHz}$ in 1993 as well [19]. However, both cases did not achieve particle acceleration. In early 2000, INFN-LNL (Italy) designed and fabricated two 81-MHz 4-rod RFQs with the cooperation of ANL, and these two SC-RFQs succeeded in accelerating heavy ions with SC-RFQ in the PIAVE beam line at INFN LNL and have been in operation till now [20].

A prior research [21] has conducted a beam simulation similar to the one described in this research. The preceding study evaluated $Q_{\mathrm{rf}}$ and $Q_{\mathrm{b}}$ with a beam simulation of 350$\mathrm{MHz}$ four-vane SC-RFQ that the proton beams $(50 \mathrm{~mA}$, $50 \mathrm{keV}, 0.0075 \mathrm{~cm} \mathrm{mrad})$ at RFQ entrance position are accelerated to $2.5 \mathrm{MeV}$ at the RFQ exit. PARMTEQ was used as a simulation software to design the RFQ structure and evaluate the beam dynamics. Moreover, the surface rf resistance of a SC-RFQ was assumed to be $100 \mathrm{n} \Omega$. The number of MC particles was selected as 10,000. Nonetheless, the differences between the preceding study and this study are listed herein for reference. In this study, a newer simulation software RFQGen was used to design the RFQ structure and evaluate the beam dynamics, and we considered a more realistic situation regarding the BNCT to produce neutrons with proton/deuteron beams $(30 \mathrm{~mA}$, $50 \mathrm{keV}, 0.02 \mathrm{~cm} \mathrm{mrad}$ ) accelerated at $2.5 \mathrm{MeV}$ (for the lithium target) or $5 \mathrm{MeV}$ (for the beryllium target). In addition, the current study adopted the number of MC particles as 100,000 to suppress the statistical errors as compared to the preceding study. Moreover, the preceding study did not design a LEBT that could separate and eliminate molecular ions and poor emittance beams arising from the ion source. Additionally, our study considered a more realistic rf surface resistance that was determined by the semiempirical formula for the $\mathrm{Nb}$ BCS resistance in Eq. (A5) and the residual resistance of $43 \mathrm{n} \Omega$ obtained by the vertical test measurement of the CH-DTL used at TE2 1 mode [14].

$E_{p}$ is a highly important parameter that can influence the suppressing of the heat generated in a cryomodule, and consequently shortening the RFQ length. However, the extent to which $E_{p}$ can be raised without degrading the Q-value depends on the smoothness and cleanliness of the SRF cavities. According to the vertical test results of elliptical cavities evaluated at KEK, the degradation of the Q-value was not confirmed to occur until $E_{p}=40 \mathrm{MV} / \mathrm{m}$ for most cases. On the other hand, it is not assured that SRF cavities with an RFQ-like structure exhibit such trends. A previous study [22] conducted the
Q-E measurement in TE21 mode using the SRF cavity with a 6.5-cm four-vane structure similar to that of the RFQ. In this measurement, the degradation of $\mathrm{Q}$ did not occur up to $E_{p}=100 \mathrm{MV} / \mathrm{m}$. Contrarily, the Q-E measurement results of 4-rod RFQ at $81 \mathrm{MHz}$ in INFN LNL at the PIAVE beam line revealed the degradation of $\mathrm{Q}$ at around $E_{p}=25 \mathrm{MV} / \mathrm{m}$ owing to field emission [20]. However, the size of the cavity for the system at INFN was significantly larger than that of the cavity assumed in this study; in addition, the RFQ structure was four-rod instead of four-vane, and the Q-E measurement was conducted 20 years ago. Thus, we need to ascertain the extent to which the current SRF surface treatment technique can update $E_{p}$ by using the four-vane SC-RFQ prototype. Therefore, the establishment of a fabrication method to ensure that the four-vane SC-RFQ achieves $E_{p}>40 \mathrm{MV} / \mathrm{m}$ is essential for future progress.

Generally, hydrogen/deuterium gas arising from the ion source enters the RFQ through the LEBT. J-PARC RFQ adopts a dedicated differential exhaust pumping system to achieve the space charge neutralization effect of $90 \%$ at the degree of vacuum of $2.8 \times 10^{-5} \mathrm{~Pa}$ at the RFQ entrance position [23], which is two orders of magnitude worse than that of the SRF cavity during an ordinal particle accelerator operation. The performance may deteriorate owing to the adsorption of gas into the inner surface of the SC-RFQ as the vacuum level is the same level as J-PARC at the present time. Thus, SC-BNCT should adopt a more powerful differential exhaust pumping system installed in front of the cryomodule than the one operating at J-PARC RFQ. In addition, this paper puts forward the following ideas to improve the degree of vacuum at the RFQ entrance: (i) increasing the diameter of the beam pipe of LEBT to dilute hydrogen/deuteron gas flowing through the LEBT. (ii) cooling the beam pipe of LEBT with a coolant such as liquid nitrogen to make LEBT play a role similar to cryopump. (iii) increasing the number of orifices at positions where the beams do not intersect to worse the hydrogen/deuteron gas conductivity in LEBT. Future progress should establish an experimental technique that can effectively improve the degree of vacuum at the RFQ entrance and clarify the influence of the degree of vacuum on the cavity performance.

The performance of RFQ suffers from the influence of fabrication errors due to machining an RFQ vane and aligning the RFQ body. If additional beam-induced heat generation due to fabrication errors is non-negligible, the cooling capacity of $100 \mathrm{~W}$ of the commercially available helium refrigerator assumed in this study has to be increased further. As a result, the benefits of the SCBNCT might be nullified by the additional preparation cost of the new refrigerator. In general, the influence of fabrication errors can be compensated by tuning the intervane voltage of the RFQ $(V)$, and the residual components that cannot be completely eliminated appears 
as the fluctuation of the intervane voltage $(\Delta V)$. For example, the J-PARC RFQ has achieved $\Delta V / V$ less than $2 \%$ after tuning intervane voltage components. Meanwhile, there is no reported example for the SC-RFQ until now. Thus, the effect of $\Delta V / V$ on the performance of SC-BNCT should be evaluated. Fortunately, a comprehensive error study of the RFQ already exists to evaluate the effect of $\Delta V / V$ on beam transmission rate of the RFQ in literature [24]. Here, the error analysis of the RFQ of European Spallation Source (ESS) is performed assuming a proton beam $(75 \mathrm{keV}, 70 \mathrm{~mA}, 0.02 \mathrm{~cm} \mathrm{mrad})$ is accelerated up to 3.62 MeV. Beam simulations for 1000 different ESS RFQs, randomly generated with $\Delta V_{\max } / V$ in $[1 ; 2 ; 3 ; 4 ; 5] \%$, have been performed and the obtained results are compared. As a result, a statistical distribution for the variation of beam transmission rate through ESS RFQ depending on $\Delta V_{\max } / V$ was generated. From here, we can evaluate the beam transmission rate corresponding to the mean value and the lower value of $90 \%$ confidence level (hereinafter referred to as mean and 90\% C.L., respectively). Using beam losses in the RFQ caused by $\Delta V / V$ determined in literature [24], and using the obtained results between the beam loss rate and $Q_{\mathrm{b}}$ evaluated from the beam simulation conducted in this study for proton $2.5 \mathrm{MeV}(7.6 \mathrm{~W} / 0.06 \%$ for $E_{p}=35 \mathrm{MV} / \mathrm{m}$ ), we eventually evaluated the amount of beam-induced heat generation depending on $\Delta V_{\max } /$ $V=[2 ; 3 ; 4 ; 5]$. The comparison of ac power consumption of SC-BNCT to various degrees of $\Delta V_{\max } / V$ determined in such a way is described in the following. (1) $\Delta V_{\max } / V=2 \%$ : The corresponding beam loss is $0.04 \%$ (mean) $/ 0.2 \%$ (90\% C.L.), resulting in beam-induced heat generation of $5 \mathrm{~W}$ (mean)/25 W (90\% C.L.). The additional heat amounts evaluated for the mean value is five times smaller than that of the $90 \%$ C.L. Even assuming the value of the $90 \%$ C.L., the heat generated in the cryomodule amounts to about $60-67 \mathrm{~W}$, which is within $100 \mathrm{~W}$ (i.e., the maximum cooling capacity of a commercially available helium refrigerator assumed in this study). The ratio of the ac power consumption of the SC-BNCT system to that of the conventional BNCT system is $26 \%$ (proton $2.5 \mathrm{MeV}$ )/ $27 \%$ (proton $5 \mathrm{MeV}$ ) $/ 22 \%$ (deuteron $5 \mathrm{MeV}$ ). The $\mathrm{SC}$ BNCT system remains much superior in terms of the ac power consumption. (2) $\Delta V_{\max } / V=3 \%$ : The corresponding beam loss is $0.09 \%$ (mean) $/ 0.3 \%$ ( $90 \%$ C.L.), resulting in beam-induced heat generation of $11 \mathrm{~W}$ (mean)/38 W (90\% C.L. The additional heat amount evaluated in the case for the mean value is about three times smaller than that for the $90 \%$ C.L. Even assuming the value of the $90 \%$ C.L., the heat generated in the cryomodule amounts to $73-80 \mathrm{~W}$, which is within the range of $100 \mathrm{~W}$. The ratio of the ac power consumption of the SC-BNCT system to that of the conventional BNCT system is $27 \%$ (proton $2.5 \mathrm{MeV}$ )/28\% (proton $5 \mathrm{MeV}$ )/23\% (deuteron $5 \mathrm{MeV}$ ). The SC-BNCT system remains superior in terms of the ac power consumption. (3) $\Delta V_{\max } / V=4 \%$ : The corresponding beam loss is $0.19 \%$ (mean) $/ 0.67 \%$ (90\% C.L.), resulting in beaminduced heat generation of $24 \mathrm{~W}$ (mean)/85 W (90\% C.L. The additional heat amounts generated in the case for the mean value is the same level as that for the $90 \%$ C.L. of $\Delta V_{m} a x / V=2 \%$. The total heat amounts generated in the case for the $90 \%$ C.L. is $119-126 \mathrm{~W}$, which exceeds $100 \mathrm{~W}$. Then, it is desirable to upgrade the maximum cooling capacity of a commercially available helium refrigerator assumed in this study for SC-BNCT to $200 \mathrm{~W}$ class to maintain the safety level. In this case, for simplicity, assuming that the heat removal efficiency of the refrigerator of the $200 \mathrm{~W}$ class is same as that of the $100 \mathrm{~W}$ class, the ratio of the ac power consumption of the SC-BNCT system to that of the conventional BNCT system is estimated to be $31 \%$ (proton $2.5 \mathrm{MeV}$ ) $/ 30 \%$ (proton $5 \mathrm{MeV}$ ) $/ 26 \%$ (deuteron $5 \mathrm{MeV}$ ). The $\mathrm{SC}-\mathrm{BNCT}$ system remains superior in terms of the ac power consumption. (4) $\Delta V_{\max } / V=5 \%$ : The corresponding beam loss is $0.37 \%$ (mean) $/ 2 \%$ (90\% C.L.), resulting in beam-induced heat generation of $47 \mathrm{~W}$ (mean) $/ 253 \mathrm{~W}(90 \%$ C.L. The total heat amount generated in the cryomodule for the $90 \%$ C.L. further exceeds the maximum cooling capacity of a commercially available helium refrigerator assumed in this study. It is inevitable to upgrade the cooling capacity to $600 \mathrm{~W}$ to maintain the sufficient safety level. This situation is no longer realistic because the cost for the preparation of the helium refrigerator of $600 \mathrm{~W}$ becomes drastically higher than that of the $100 \mathrm{~W}$ refrigerator initially considered in this study.

From these discussions, it can be concluded that the feasibility of the SC-BNCT system can be guaranteed if $\Delta V_{\max } / V$ is kept within $4 \%$. This value corresponds to two times looser than the value typically achieved in the tuning of the intervane voltage of NC-RFQ. We should therefore aim to satisfy the criterion in the fabrication stage for SC-BNCT.

In the following section, we will discuss how to fabricate the SC-RFQ considering the result of error analysis of tuning vane voltage described earlier. We consider the following two methods of fabricating SC-RFQ: (1) fabricating from bulk $\mathrm{Nb}$ and (2) fabricating by $\mathrm{Nb}$ sputtering. To meet the criteria shown in the aforementioned error study, it is necessary to adopt a fabrication method that can achieve high machining accuracy of the vane voltage and sufficiently suppresses the alignment error. For (1), to achieve high machining accuracy, only the vane electrode is machined from bulk niobium, and it is joined to the other parts of the base made of the bulk niobium material via electron beam welding. To achieve high alignment accuracy in this process, it is necessary to consider the effect of welding shrinkage. We also need to consider the effect of shrinkage during cooling of the SC-RFQ at $4.2 \mathrm{~K}$. Therefore, we conduct cooling tests with prototypes made using several different electron beam welding conditions to search for conditions under which mechanical tolerances 
$<100 \mu \mathrm{m}$ can be achieved. Therefore, we aim to reduce the tuning error of the vane voltage of the SC-RFQ to within $4 \%$. The merit of this fabrication method is that the cavity is made of bulk niobium so that a stable performance can be achieved even in high E-field. Unlike elliptical cavities, the performance of the superconducting RFQ is not limited by the quench field. However, there is room to significantly improve the accelerating field provided that the cavity performance is not limited by field emission. Meanwhile, as in method (2), it is possible to fabricate SC-RFQs by sputtering the $\mathrm{Nb}$ film inside a copper RFQ. The merit of this method is that it can achieve the same level of high machining and alignment accuracy as the normal-conductive RFQ. However, this method requires the niobium coating process to fabricate SC-RFQs.

Hereinafter, we compare the cost and area required by the SC-BNCT to those of the conventional one. Here, the cost and area are estimated from actual examples of the existing J-PARC RFQ and compact ERL operated at KEK. Figure 15 shows the result of the comparison calculated in such a manner. In this estimation, the price of the water cooling system of the conventional BNCT system is estimated to be about three times that of the J-PARC one because the amount of heat generated during operation of $\mathrm{i}$-BNCT is expected to be three times higher than that of J-PARC. Although this is only a comparison based on existing accelerator systems, it can be observed that the price of the superconducting BNCT system is not higher than that of the normal-conducting one. It is also clear that the superconducting BNCT system is much more compact than the normal-conducting one. However, because there is no superconducting BNCT system thus far, we cannot conclude the exact cost of the system at the present time. It is necessary to continue to make effort to fabricate cryomodules at low cost in an actual manufacturing stage. The superconducting BNCT system assumed in this study mainly consists of one cryomodule and one liquid helium refrigerator and three to five $20 \mathrm{~kW}$ rf amplifiers. All of them are commercially available and are expected to be handled without special proficient skill or technique.

Our discussion was mainly focused on a BNCT system with a rf-linac based neutron source consisting only of

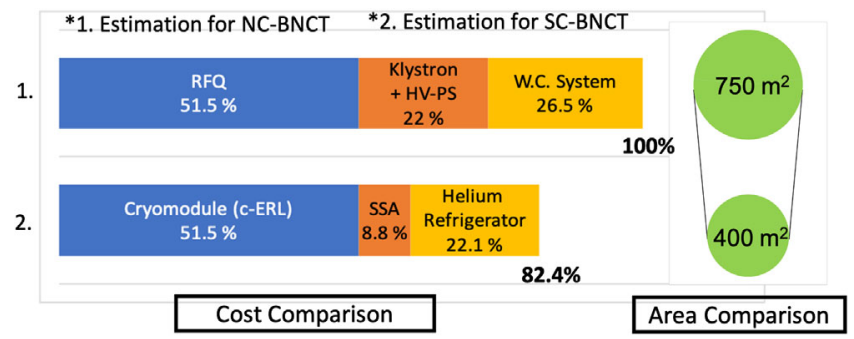

FIG. 15. Relative ratio of the estimation of NC-BNCT to that of SC-BNCT. Histogram and circle graph represent the cost and the area estimated by J-PARC and c-ERL at KEK, respectively.
RFQ. However, there is another BNCT system with a rflinac consisting of DTL along with RFQ. In the following, we will compare the ac power consumption, cost, and required area of the BNCT system adopting the RFQ-DTL combination to those of SC-BNCT that adopt only SCRFQ. For concrete example, i-BNCT employs a $1.2 \mathrm{MW}$ klystron and a $324 \mathrm{MHz}$ RFQ and DTL, aiming to achieve a proton beam current of 5-10 $\mathrm{mA}$ and beam energy of $8 \mathrm{MeV}$ in duty $10 \%-20 \%$. However, it is generally difficult to control the two accelerators with different Q-values using a single rf source, especially for medical personnel without proficient skill on an accelerator. In this estimation, we will consider two klystrons with an output power of $600 \mathrm{~kW}$ as rf sources for a BNCT system using RFQ and DTL. Based on this precondition, comparison of cost and area are summarized in Fig. 16. In this paper, we assume that BNCT operates for 10 hours per day in a hospital and the power efficiency of a rf source is $50 \%$. Hence, the ac power consumption of the BNCT system using RFQ and DTL is estimated to be $2.4-4.8 \mathrm{MWh} /$ day. This value is comparable to or greater than twice that of the ac power consumption of the SC-BNCT system evaluated in this paper. Additionally, Fig. 16 shows that the estimated cost of the BNCT system using RFQ and DTL is more than twice that of the SC-BNCT system.

In general, ion sources have output instability that cause fluctuations in the emittance of incident beams to the RFQ entrance by $\sim 10 \%$. This study adopted the beam optics of the LEBT design to transmit only the good emittance beams equivalent to or better than $0.02 \mathrm{~cm} \mathrm{mrad}$. Thus, the fluctuation of the beam emittance reflects that of the beam current at the RFQ entrance position. In addition, the variation in the beam current according to the $10 \%$ emittance instability was evaluated by performing a LEBT proton/deuteron beam simulation, assuming an incident beam with an intensity of $50 \mathrm{keV}, 30 \mathrm{~mA}$ and emittances of $0.02,0.03,0.04,0.06,0.08,0.10$, and $0.20 \mathrm{~cm} \mathrm{mrad}$. Figures 17 and 18 depict the proton/ deuteron beam transmission rate as a function of incident beam emittance evaluated by the simulation. The horizontal and vertical axes represent the beam emittance and beam

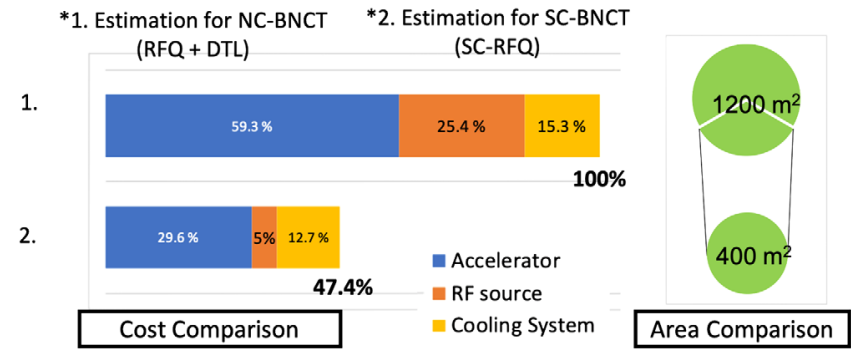

FIG. 16. Relative ratio of the estimation of NC-BNCT $(\mathrm{RFQ}+\mathrm{DTL})$ to that of SC-BNCT. Histogram and circle graph represent the cost and the area estimated by J-PARC and c-ERL at KEK, respectively. 


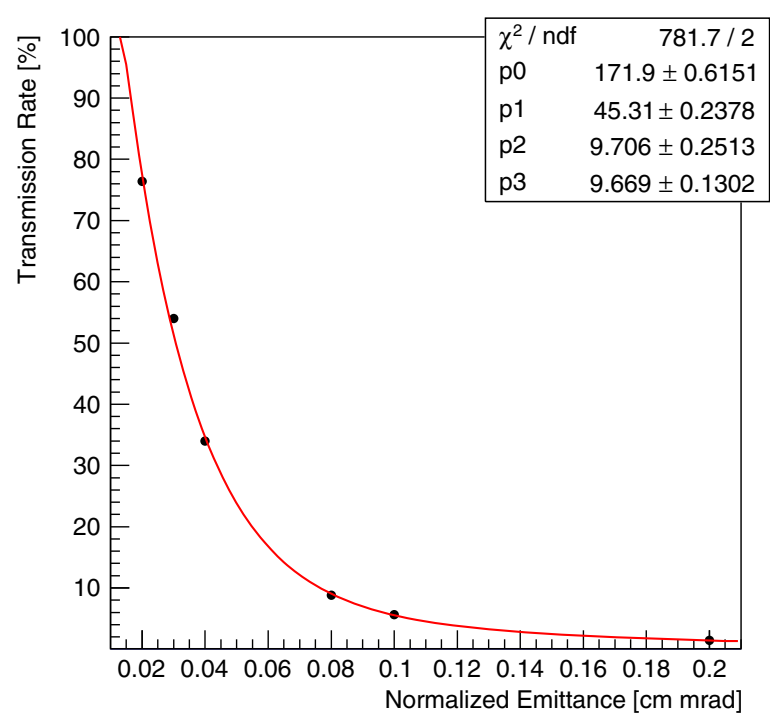

FIG. 17. Proton beam transmission rate as a function of normalized emittance. The horizontal and vertical axes represent the normalized emittance assumed in the simulation and the proton beam transmission rate through LEBT was evaluated by RFQGen simulation. The red curve depicts the fitting result with a function of $f(x)=p_{0} \exp \left(-p_{1} x\right)+p_{2} \exp \left(-p_{3} x\right)$.

transmission rate at the RFQ entrance position, respectively. Each circle denotes the data point obtained from simulation. The red line depicts the fitting curve of the function $f(x): \quad f(x)=p_{0} \exp \left(-p_{1} x\right)+p_{2} \exp \left(-p_{3} x\right)$. Thus, we can evaluate the change in the beam transmission rate caused by the instability of the beam emittance with this fitting result. For instance, we considered an average

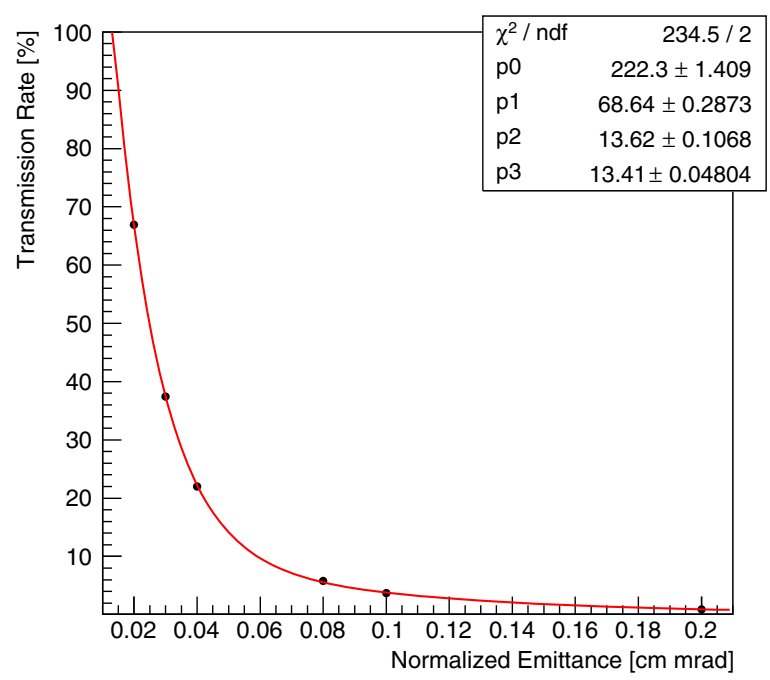

FIG. 18. Deuteron beam transmission rate as a function of normalized emittance. The horizontal and vertical axes represent the normalized emittance assumed in the simulation and the deuteron beam transmission rate through LEBT was evaluated by RFQGen simulation. The red curve depicts the fitting result with a function of $f(x)=p_{0} \exp \left(-p_{1} x\right)+p_{2} \exp \left(-p_{3} x\right)$. value of the beam emittance of $0.025 \mathrm{~cm}$ mrad (same as IFMIF) and the corresponding fluctuation as 10\% (as practical). In this case, the corresponding fluctuation of the beam transmission rate was estimated to be $10 \%-11 \%$ (for proton) $/ 13 \%-16 \%$ (for deuteron). Consequently, the influence of the emittance fluctuation was not as large as the effective beam current decreased.

\section{CONCLUSION}

This study evaluated the feasibility of applying a Nb SRF cavity on a rf linac-based neutron source for BNCT, considering that a 4.2-K SC-RFQ was used to accelerate the proton/deuteron beams onto a beryllium/lithium target via ${ }^{7} \mathrm{Li}(\mathrm{p}, \mathrm{n}){ }^{7} \mathrm{Be},{ }^{9} \mathrm{Be}(\mathrm{p}, \mathrm{n}){ }^{9} \mathrm{~B}$ or ${ }^{9} \mathrm{Be}(\mathrm{d}, \mathrm{n}){ }^{10} \mathrm{~B}$ for the neutron production of BNCT. The beam parameters used in this study included: a beam energy of $2.5 \mathrm{MeV}$ (for Li target)/ $5 \mathrm{MeV}$ (for Be target), an ion source current $(50 \mathrm{keV}, \mathrm{CW}$ $30 \mathrm{~mA}$ ), a criterion of beam emittance $0.02 \mathrm{~cm} \mathrm{mrad}$, and a resonance frequency $325 \mathrm{MHz}$ (for proton)/162.5 MHz (for deuteron). Moreover, certain tasks remained to be fulfilled to ensure the practical application of SRF cavities: the heat generated in a cryomodule must be suppressed to lower than the cooling capacity of a commercially available $4.2-\mathrm{K}$ helium refrigerator, and the ac power consumption for a superconducting BNCT system must be superior to that of a conventional BNCT system. In addition, the size of the SC-RFQ can be decreased to be equal to or smaller than that of the existing normal-conducting RFQs. Thus, the aforementioned preconditions were assumed in this study to evaluate the amount of heat generated in a cryomodule, RFQ length, and ac power consumption by performing deuteron/proton beam simulations using a RFQ structure that was designed to achieve a transmission rate greater than $99.8 \%$. The ion sources listed in Table II, LEBT in Table III, and Table IV, RFQ design per Table V, and 100,000 MC particles were used in this study.

The peak surface E-field $\left(E_{p}\right)$ and minimum radial aperture $\left(a_{0}\right)$ determined from the RFQGen tend to be

TABLE XI. Conceptional design of SC-RFQ for BNCT with a proton acceleration of $2.5 \mathrm{MeV}$.

\begin{tabular}{lcc}
\hline \hline Energy range & $0.05-2.5$ & $\mathrm{MeV}$ \\
\hline p current at ion source & 30 & $\mathrm{~mA}$ \\
Transmission (LEBT) & 76.4 & $\%$ \\
Transmission (RFQ) & 99.9 & $\%$ \\
Material target & Lithium & \\
Peak surface E-field & 40.78 & $\mathrm{MV} / \mathrm{m}$ \\
RMS norm emittance & 0.02 & $\mathrm{~cm} \mathrm{mrad}$ \\
Final modulation factor & 2.0502 & \\
Final synchronous phase & -35 & $\mathrm{deg}$ \\
RFQ length & 296.6 & $\mathrm{~cm}$ \\
Beam power & 57.3 & $\mathrm{~kW}$ \\
RF power dissipation & 7.9 & $\mathrm{~W}$ \\
Lost beam power at RFQ & 6.3 & $\mathrm{~W}$ \\
\hline \hline
\end{tabular}




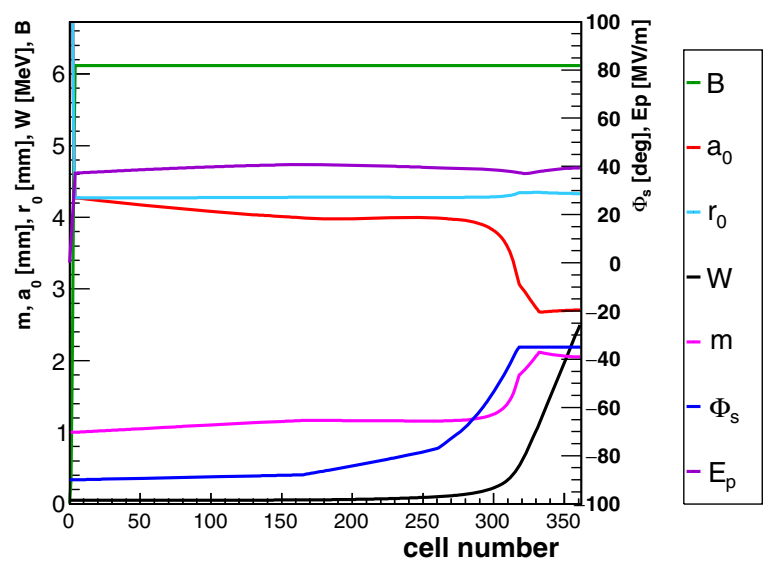

FIG. 19. Cell parameters for conceptual RFQ design of the 2.5$\mathrm{MeV}$ proton accelerator proposed in this paper.

slightly lower than the original input value set. The beam current at the RFQ exit was $23 \mathrm{~mA}$ (for protons) and $20 \mathrm{~mA}$ (for deuteron), which were sufficient to produce the number of neutrons required for the BNCT. Moreover, the results of the beam simulations revealed that the surface heat depends on $E_{p}$, as shown in Figs. 12 and 13. Thus, if $E_{p}>$ $35 \mathrm{MV} / \mathrm{m}$ (for proton) and $E_{p}>30 \mathrm{MV} / \mathrm{m}$ (for deuteron) are satisfied, the surface heat can be maintained within the range of $100 \mathrm{~W}$, which is the typical cooling capacity of a commercially available helium refrigerator. In addition, the dependence of the RFQ length on $E_{p}$ is presented in Fig. 14. The RFQ length for the deuteron accelerator can be shortened to $66 \%$ as compared to the IFMIF RFQ at $E_{p} \sim 30 \mathrm{MV} / \mathrm{m}$, and the RFQ length for a proton accelerator at $E_{p} \sim 40 \mathrm{MV} / \mathrm{m}$ is similar to that of the existing RFQs. Furthermore, the ac power consumption required for the SC-BNCT was determined from the input power of the rf source and the operational heat-removal power of the refrigerator. In this study, the power efficiency of the rf source was $50 \%$, the cooling efficiency of the surface heat was $0.6 \mathrm{~kW} / \mathrm{W}$, and the penetration heat to the cryomodule

TABLE XII. Conceptual design of SC-RFQ for BNCT with a proton acceleration of $5 \mathrm{MeV}$.

\begin{tabular}{lcc}
\hline \hline Energy range & $0.05-5$ & $\mathrm{MeV}$ \\
\hline p current at ion source & 30 & $\mathrm{~mA}$ \\
Transmission (LEBT) & 76.4 & $\%$ \\
Transmission (RFQ) & 99.9 & $\%$ \\
Material target & Beryllium & \\
Peak surface field & 40.81 & $\mathrm{MV} / \mathrm{m}$ \\
RMS norm emittance & 0.02 & $\mathrm{~cm} \mathrm{mrad}$ \\
Final modulation factor & 2.0349 & \\
Final synchronous phase & -35 & $\mathrm{deg}$ \\
RFQ length & 508.3 & $\mathrm{~cm}$ \\
Beam power & 114.5 & $\mathrm{~kW}$ \\
RF power dissipation & 13.6 & $\mathrm{~W}$ \\
Lost beam power at RFQ & 3.2 & $\mathrm{~W}$ \\
\hline \hline
\end{tabular}

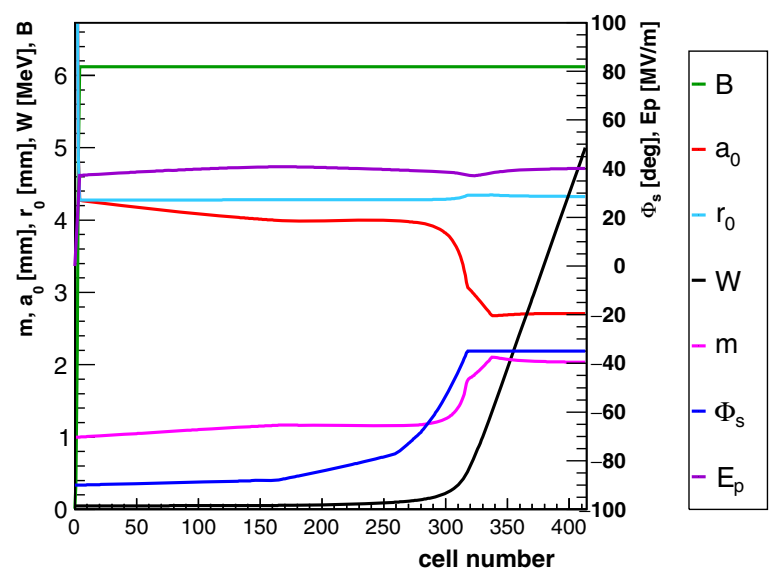

FIG. 20. Cell parameters for conceptual RFQ design of the 5$\mathrm{MeV}$ proton accelerator proposed in this paper.

from the outside and its cooling efficiencies were $20 \mathrm{~W}$ and $1 \mathrm{~kW} / \mathrm{W}$, respectively. Therefore, the ac power consumption of the SC-BNCT system was numerically evaluated based on these conditions. Consequently, the ac power consumption in the SC-BNCT system was reduced to approximately one-fourth as compared with that of a conventional BNCT. Lastly, the maximum surface electric fields of $E_{p}>30 \mathrm{MV} / \mathrm{m}$ (for deuteron) and $E_{p}>$ $40 \mathrm{MV} / \mathrm{m}$ (for protons) imparted compactness to the SC-BNCT system and reduced the ac power consumption to approximately one-fourth of that for a conventional BNCT system, which provides a foundation for the application of the SRF $\mathrm{Nb}$ cavity to the rf linac-based neutron source for BNCT.

The design of the SC-RFQ for BNCT proposed and evaluated in this study is presented herein. In a conservative approach, we assumed the weakest peak surface E-field that could guarantee the feasibility of the SC-BNCT system. The design specification of SC-RFQ for the BNCT system in which proton beams of $2.5 \mathrm{MeV}$ are injected into the lithium target for neutron production is presented in

TABLE XIII. Conceptual design of SC-RFQ for BNCT with a deuteron acceleration of $5 \mathrm{MeV}$.

\begin{tabular}{lcc}
\hline \hline Energy range & $0.05-5$ & $\mathrm{MeV}$ \\
\hline d current at Ion source & 30 & $\mathrm{~mA}$ \\
Transmission (LEBT) & 66.9 & $\%$ \\
Transmission (RFQ) & 99.8 & $\%$ \\
Material target & Beryllium & \\
Peak surface field & 31.8 & $\mathrm{MV} / \mathrm{m}$ \\
RMS norm emittance & 0.02 & $\mathrm{~cm} \mathrm{mrad}$ \\
Final modulation factor & 2.0358 & \\
Final synchronous phase & -35 & $\mathrm{deg}$ \\
RFQ length & 638.2 & $\mathrm{~cm}$ \\
Beam power & 100.3 & $\mathrm{~kW}$ \\
rf power dissipation & 8.3 & $\mathrm{~W}$ \\
Lost beam power at RFQ & 13.8 & $\mathrm{~W}$ \\
\hline \hline
\end{tabular}




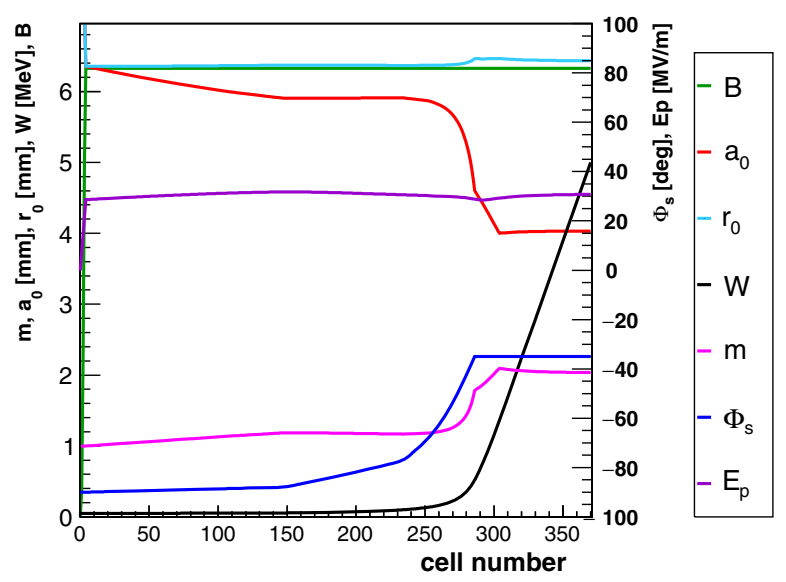

FIG. 21. Cell parameters for conceptual RFQ design of the $5-\mathrm{MeV}$ deuteron accelerator proposed in this paper.

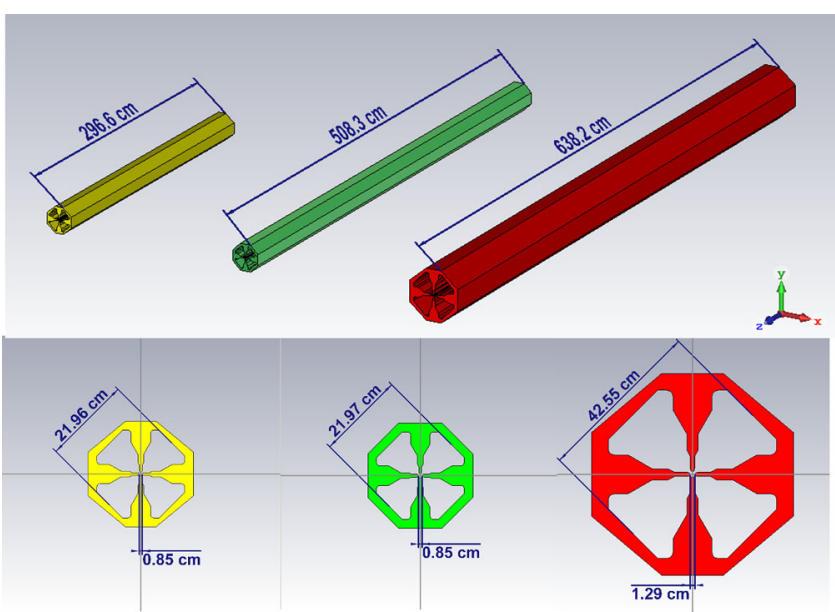

FIG. 22. Comparison of 3D models for the conceptual design of SC-RFQ for BNCT. Yellow: $2.5 \mathrm{MeV}$ proton, blue: $5 \mathrm{MeV}$ proton, red: $5 \mathrm{MeV}$ deuteron.

Table XI and Fig. 19, that for proton beams of $5 \mathrm{MeV}$ are injected into the beryllium target is presented in Table XII and Fig. 20, and that for deuteron beams of $5 \mathrm{MeV}$ are injected into the beryllium target is presented in Table XIII and Fig. 21. Furthermore, the 3D model shapes of the superconducting RFQ based on each design specification are illustrated in Fig. 22 (yellow: $2.5 \mathrm{MeV}$ proton, blue: $5 \mathrm{MeV}$ proton, Red: $5 \mathrm{MeV}$ deuteron). Thus, the design specifications of the accelerator components for an accelerator-based neutron source for the SC-BNCT system proposed in this study are summarized.

\section{ACKNOWLEDGMENTS}

We are deeply grateful to Fujio Naito, of High Energy Accelerator Research Organization, for incisive comments and meaningful discussion on the concept of SC-BNCT.

\section{APPENDIX: POWER LOSS OF A FOUR VANE RFQ}

According to previous studies [25], the rf power lossequivalent to the ohmic loss - of a four-vane RFQ derived from the lumped circuit model of a four-vane cloverleaf geometry can be expressed as follows:

$$
P_{\mathrm{RFQ}}=\frac{1}{4} \sqrt{\frac{4+3 \pi}{\mu_{0}}} R_{s} \omega C_{l}^{\frac{3}{2}} V^{2} L,
$$

where $P_{\mathrm{RFQ}}$ represents the ohmic loss of a four-vane RFQ owing to the rf surface resistance calculated from the lumped circuit model, $\mu$ is the permeability in vacuum, $R_{s}$ represents the rf surface resistance of an RFQ, $C_{l}$ is the total capacitance of a RFQ per unit length, $\omega$ represents the resonant angular frequency of a RFQ, $V$ represents an intervane voltage applied in the RFQ, and $L$ represents the length of the RFQ.

The $\mathrm{rf}$ resistance of the surface coated with copper, $R_{\mathrm{Cu}}$, is expressed by the following formula:

$$
R_{\mathrm{Cu}}=\sqrt{\frac{\omega \mu_{0}}{2 \sigma}}
$$

where $\sigma$ is the electrical conductivity of pure copper, and the permeability of the copper was regarded as that in vacuum.

Substituting Eq. (A2) for Eq. (A1), the following equation can be derived for the ohmic loss of a RFQ coated with copper thick films, $P_{\mathrm{NC}-\mathrm{RFQ}}$ :

$$
P_{\mathrm{NC}-\mathrm{RFQ}}=\sqrt{\left(\frac{4+3 \pi}{32 \sigma}\right)}\left(\omega C_{l}\right)^{\frac{3}{2}} V^{2} L
$$

Equation (A3) has the single free parameter $C_{l}$, which can be estimated by calculating the electromagnetic code SUPERFISH considering a four-vane cloverleaf geometry. Although the calculation result showed that $C_{l}$ is a weak function of the vane radius, the typical values are known to be approximately $120 \times 10^{-12} \mathrm{~F} / \mathrm{m}$ [25]. In this study, the value of $C_{l}$ was assumed to be constant at $120 \times 10^{-12} \mathrm{~F} / \mathrm{m}$, independent of the resonant frequency.

On the contrary, the rf surface resistance of a SRF cavity made of pure bulk $\mathrm{Nb}$ can be expressed as follows:

$$
R_{\mathrm{Nb}}=R_{\mathrm{BCS}}+R_{\mathrm{res}},
$$

where $R_{\mathrm{BCS}}$ denotes the BCS resistance of pure bulk niobium, and $R_{\text {res }}$ denotes the residual resistance of bulk niobium.

The temperature and frequency dependence of $R_{\mathrm{BCS}}$ is expressed by the following semiempirical formula [26]: 


$$
R_{\mathrm{BCS}}=\left(2 \times 10^{-4} \frac{1}{T}\left(\frac{f}{1.5}\right)^{2} \exp \left(\frac{-17.67}{T}\right)\right)
$$

For example, $R_{\mathrm{BCS}}$ at $4.2 \mathrm{~K}$ of $162.5 \mathrm{MHz}$ is $8 \mathrm{n} \Omega$, that of $325 \mathrm{MHz}$ is $33 \mathrm{n} \Omega$, and that of $1.3 \mathrm{GHz}$ is $533 \mathrm{n} \Omega$.

Thus, the ohmic loss of an SC-RFQ made of bulk niobium due to $\mathrm{BCS}$ resistance $P_{\mathrm{SC}-\mathrm{RFQ}}$ can be expressed by the following formula:

$$
P_{\mathrm{SC}-\mathrm{RFQ}}=\frac{R_{\mathrm{BCS}}}{R_{\mathrm{Cu}}} P_{\mathrm{NC}-\mathrm{RFQ}}
$$

[1] National Cancer Center at Japan, Linear accelerator based BNCT system enabled to be installed in a hospital, March 2016, Available at: https://www.ncc.go.jp/jp/information/ pr_release/2016/0301/press_release_20160301_shiryo02 .pdf (Accessed: 6 September 2020).

[2] F. Naito, S. Anami, Z. Fang, S. Fujikura, and K. Futatsukawa, Beam Commissioning of the i-BNCT LINAC, in Proceedings of LINAC2016, East Lansing, MI, USA (JACoW, Geneva, Switzerland, 2016), p. 760-762.

[3] Y. Kiyanagi, Accelerator driven neutron sources, J. Part. Accel. Soc. Jpn. 13, 192 (2016), http://www.pasj.jp/kaishi/ cgi-bin/kasokuki.cgi?articles\%2F13\%2Fp192-197.pdf.

[4] K. Hosoyama, Cryogenic technology for superconducting accelerators, Rev. Accel. Sci. Techol. 05, 205 (2012).

[5] M. Busch, M. Basten, T. Conrad, P. Müller, H. Podlech, M. Schwarz, W. A. Barth, F. D. Dziuba, and M. Miski-Oglu, Overview on SC CH-cavity development, in Proceedings of IPAC2019, Melbourne, Australia (JACoW, Geneva, 2019), p. 2822-2825.

[6] JAERI/KEK Joint Project Team Accelerator Group, Accelerator technical design report for J-PARC, KEK-Report 2002-13 and JAERI-TECH 2003-044 (2003).

[7] https://lmytechnology.com.

[8] L-Series Helium Liquefiers, https://linde-kryotechnik .ch/en/products/helium-liquefiers/l-series/ (Accessed: 6 September 2020).

[9] G. Bisoffi, V. Andreev, G. P. Bezzon, F. Chiurlotto, A. Lombardi, and A. M. Porcellato, Completion of the first superconducting RFQ at INFN-LNL, in Proceedings of SRF1999, Santa Fe, NM, USA (JACoW, Geneva, Switzerland, 1999), p. 367-371.

[10] S. Posen and D. L. Hall, $\mathrm{Nb}_{3} \mathrm{Sn}$ superconducting radio frequency cavities: fabrication, results, properties, and prospects, Supercond. Sci. Technol. 30, 033004 (2017).

[11] A. Grassellino, A. Romanenko, D. Sergatskov, O. Melnychuk, Y. Trenikhina, A. Crawford, A. Rowe, M. Wong, T. Khabiboulline, and F. Barkov, Nitrogen and argon doping of niobium for superconducting radio frequency cavities: a pathway to highly efficient accelerating structures, Supercond. Sci. Technol. 26, 102001 (2013).

[12] J. Billen and L. Young, Poisson superfish, Los Alamos National Laboratory, Report No. LA-UR-96-1834, 1996.
[13] Y. Kondo, H. Asano, E. Chishiro, K. Hirano, T. Ishiyama, T. Itou, Y. Kawane, N. Kikuzawa, S. Meigo, A. Miura et al., A $3 \mathrm{MeV}$ LINAC for development of accelerator components at J-PARC, in Proceedings of LINAC2016, East Lansing, MI, USA (JACoW, Geneva, Switzerland, 2016), p. 298-300.

[14] U. Ratzinger, G. Clemente, C. Commenda, and H. Liebermann, A $70 \mathrm{MeV}$ proton linac for the FAIR facility based on CH cavities, in Proceedings of LINAC 2006, Knoxville, Tennessee USA (JACoW, Geneva, Switzerland, 2006), p. 526-530.

[15] E. Fagotti, L. Antoniazzi, L. Bellan, D. Bortolato, M. Comunian, A. Facco, M. Giacchini, F. Grespan, M. Montis, A. Palmieri et al., Beam commissioning of the IFMIF EVEDA very high power RFQ, in Proceedings of IPAC2018, Vancouver, BC, Canada (JACoW, Geneva, 2018), pp. 2902-2907.

[16] A. Aleksandrov, B. Cathey, S. Cousineau, M. Crofford, and B. Han, Commissioning of the new SNS RFQ and $2.5 \mathrm{MeV}$ beam test facility, in Proceedings of IPAC2017, Copenhagen, Denmark (JACoW, Geneva, 2017), pp. 2438-2440.

[17] A. Pisent, E. Fagotti, and P. Colautti, MUNES a compact neutron source for BNCT and radioactive wastes characterization, in Proceedings of LINAC2014, Geneva, Switzerland (JACoW, Geneva, Switzerland, 2014), 261263.

[18] K. W. Shepard, W. L. Kennedy, and K. R. Crandall, Construction of a superconducting RFQ structure, in Proceedings of International Conference on Particle Accelerators, Washington, DC, USA (IEEE, New York, 1993), p. 10421044, Vol. 2.

[19] T. P. Wangler, A. G. Cimabue, J. Merson, R. S. Mills, R. L. Wood, and L. M. Young, Superconducting RFQ linear accelerator, Nucl. Instrum. Methods Phys. Res., Sect. B 79, 718 (1993).

[20] G. Bisoffi, Superconducting radiofrequency quadrupoles, in Proceedings of the 10th workshop on of superconductivity, Tsukuba, Japan (JACoW, Geneva, Switzerland, 2001), p. 123-131.

[21] T. P. Wangler, A. G. Cimabue, J. Merson, R. S. Mills, R. L. Wood, and L. M. Young, Superconducting RFQ development at Los Alamos, in Proceedings of the 1992 Linear Accelerator Conference, Ottawa, Ontario, Canada (Atomic Energy of Canada Limited, Chalk River Laboratories, Ontario, Canada, 1992), p. 627-629.

[22] J. R. Delayen and K. W. Shepard, Tests of a superconducting rf quadrupole device, Appl. Phys. Lett. 57, 514 (1990).

[23] A. Ueno, J-PARC $\mathrm{H}^{-}$ion source and space-charge neutralized LEBT for 100-mA high energy and high duty factor LINACs, Rev. Sci. Instrum. 91, 033312 (2020).

[24] A. Ponton, Y. I. Levinsen, and E. Sargsyan, Voltage error studies in the ESS RFQ, in Proceedings of IPAC2016, Busan, Korea (JACoW, Geneva, 2016).

[25] T. P. Wangler, RF Linear Accelerators (Wiley \& Sons, New York, 2008).

[26] H. Padamsee, J. Knobloch, and T. Hays, RF Superconductivity for Accelerators (J. Wiley \& Sons, New York, 1998). 